\title{
Pre-Hospital Care Management of a Potential Spinal Cord Injured Patient: A Systematic Review of the Literature and Evidence-Based Guidelines
}

\author{
Henry Ahn, Jeffrey Singh, ${ }^{2}$ Avery Nathens, ${ }^{3}$ Russell D. MacDonald, ${ }^{4}$ Andrew Travers, ${ }^{5}$ \\ John Tallon, ${ }^{6}$ Michael G. Fehlings, ${ }^{1}$ and Albert Yee ${ }^{1}$
}

\begin{abstract}
An interdisciplinary expert panel of medical and surgical specialists involved in the management of patients with potential spinal cord injuries (SCI) was assembled. Four key questions were created that were of significant interest. These were: (1) what is the optimal type and duration of pre-hospital spinal immobilization in patients with acute SCI?; (2) during airway manipulation in the pre-hospital setting, what is the ideal method of spinal immobilization?; (3) what is the impact of pre-hospital transport time to definitive care on the outcomes of patients with acute spinal cord injury?; and (4) what is the role of pre-hospital care providers in cervical spine clearance and immobilization? A systematic review utilizing multiple databases was performed to determine the current evidence about the specific questions, and each article was independently reviewed and assessed by two reviewers based on inclusion and exclusion criteria. Guidelines were then created related to the questions by a national Canadian expert panel using the Delphi method for reviewing the evidence-based guidelines about each question. Recommendations about the key questions included: the pre-hospital immobilization of patients using a cervical collar, head immobilization, and a spinal board; utilization of padded boards or inflatable bean bag boards to reduce pressure; transfer of patients off of spine boards as soon as feasible, including transfer of patients off spinal boards while awaiting transfer from one hospital institution to another hospital center for definitive care; inclusion of manual in-line cervical spine traction for airway management in patients requiring intubation in the pre-hospital setting; transport of patients with acute traumatic SCI to the definitive hospital center for care within $24 \mathrm{~h}$ of injury; and training of emergency medical personnel in the pre-hospital setting to apply criteria to clear patients of cervical spinal injuries, and immobilize patients suspected of having cervical spinal injury.
\end{abstract}

Key words: pre-hospital care; spinal cord injury; systematic review

\section{Introduction}

G REAT CARE MUST BE TAKEN when providing medical care To an acutely injured patient prior to arrival at hospital. About 2\% of all blunt trauma patients will have sustained a spinal cord injury, and these rates are higher in the setting of severe closed head injury (Crosby, 1992, 2006). Patients with acute spinal cord injury (SCI) are at risk of neurologic deterioration due to secondary injury to the spinal cord (Fehlings and Louw, 1996). A potential cause of secondary injury is through inadvertent manipulation of the spinal cord in the setting of an unstable spinal column injury (Crosby, 1992; Eismont et al., 2004; Fehlings and Louw, 1996; Fenstermaker, 1993). Minimizing the chances of secondary injury can be challenging in the pre-hospital setting due to the local and transport environment, a lack of resources, and heterogeneity in health care providers and their skill sets (Hauswald et al., 2000). Furthermore, treatments initiated prior to arrival in the hospital can lead to significant morbidity in other body regions, such as sacral and occipital ulcers (Cordell et al., 1995;

\footnotetext{
${ }^{1}$ Department of Surgery, ${ }^{2}$ Department of Interdepartmental Medicine, Division of Critical Care, ${ }^{3}$ Department of Surgery, Health Policy, Management and Evaluation, and ${ }^{4}$ Department of Research and Development, Ornge Transport Medicine and Division of Emergency Medicine, Department of Medicine, University of Toronto, Toronto, Ontario, Canada.

${ }^{5}$ Department of Emergency Medicine, and ${ }^{6}$ Department of Emergency Medicine, Surgery and Community Health and Epidemiology, Dalhousie University, Halifax, Nova Scotia, Canada.
} 
Davies et al., 1996; De Lorenzo et al., 1996; Hamilton and Pons, 1996; Hauswald et al., 2000; Johnson et al., 1996; Krell et al., 2006; Luscombe and Williams, 2003; Main and Lovell, 1996; Sheerin and de Frein, 2007; Walton et al., 1995). There is tremendous variation in how care is administered prior to arrival at the hospital and during transport from one hospital to another (Armitage et al., 1990; Burney et al., 1989; Flabouris, 2001). Some care models and treatments may provide patients with improved safety and reduce morbidity, and thus improve efficiency of care delivery. These variations of practice served as the impetus to perform a systematic review, in conjunction with a series of other systematic reviews related to SCI care. The purpose of this study was to provide evidence-based guidelines agreed upon by a multi-disciplinary expert panel to identify optimal care in key areas in the pre-hospital setting for patients with potential SCI.

Four questions that were of clinical relevance and that could have significant impact on patient care were determined by a multidisciplinary expert panel. These four questions posed for the systematic review were:

1. What is the optimal type and duration of spinal immobilization in patients with acute SCI?

2. During airway manipulation in the pre-hospital setting, what is the ideal method of spinal immobilization?

3. What is the impact of pre-hospital transport time to definitive care on the outcomes of patients with acute SCI?

4. What is the role of pre-hospital care providers in cervical spine clearance and immobilization?

These four questions then served as the basis of our systematic review of pre-hospital care of potential spinalcord-injured patients. The systematic review method was utilized to systematically collate and assess the literature, while minimizing bias in the assembly and interpretation of the evidence. Following an exhaustive search of the literature and collation of the identified studies into evidentiary tables, the evidence was graded and synthesized into guidelines that were refined through consensus using Delphi methodology (Hasson et al., 2000; Keeney et al., 2001; Kennedy, 2004).

\section{Methods}

Four questions of pertinent interest to a multi-disciplinary committee with expertise in the management of SCI were agreed upon to form the basis of the systematic review. Each of the four questions was amenable to a systematic review. Members of this committee included a traumatologist (Avery Nathens), three trauma triage specialists in emergency medicine (Russell MacDonald, Andrew Tavers, and John Tallon), three spine surgeons (Henry Ahn, Michael Fehlings, and Albert Yee), a critical care intensivist (Jeffrey Singh), and a fundamental scientist in SCI research (Darryl Baptiste).

A primary literature search was performed using the MEDLINE, CINAHL, Embase, and Cochrane databases. A secondary search strategy incorporated articles referred to in meta-analyses, systematic and non-systematic review articles that were found in the primary search. Additional articles that were listed in the references of retrieved original articles could be also included in the secondary search strategy. The literature searches addressed publications produced from 1966 to April 2008. Two reviewers independently selected the articles based on the inclusion and exclusion criteria, determined their level of evidence, and assessed their methodological quality according to the Downs and Black criteria (Downs and Black, 1998). Disagreement between the reviewers was reconciled by a third reviewer. All articles were directly related to prehospital care, and limited to human studies by excluding the "animal" Medical Subject Heading (MeSH). The MeSH search terms used were: "pre-hospital care," "spinal trauma," and "spinal cord injury."

Based on this methodology 66 articles were screened, and 47 were eligible based upon criteria utilized for pre-hospital care. These were scored according to the Downs and Black criteria (Downs and Black, 1998). The main results of each article and the reviewers' assessments were summarized in an evidentiary table (Table 1).

Evidence-based responses were then composed for the four questions. A panel of 5 to 10 multi-disciplinary experts (from the Solutions Network [Acute Practice Network]) using the Delphi method scrutinized the evidence-based statements for the specific questions. A level of consensus of $80 \%$ or higher was considered to be a strong agreement. Based on the level of agreement and the comments from the expert panel, recommendations were formulated for each question related to prehospital care.

\section{Findings from the systematic review}

Question 1. What is the optimal type and duration of spinal immobilization in patients with acute SCI?

In all, 25 studies were reviewed for this particular question (Chan et al., 1996; Chandler et al., 1992; Cordell et al., 1995; Cornwell et al., 2001; Davies et al., 1996, De Lorenzo et al., 1996; Gerling et al., 2000; Graziano et al., 1987; Hamilton and Pons, 1996; Hauswald et al., 2000, 1998; Huerta et al., 1987; Johnson et al., 1996; Krell et al., 2006; Luscombe and Williams, 2003; Main and Lovell, 1996; Mazolewski and Manix, 1994; Nypaver and Treloar, 1994; Peery et al., 2007; Perry et al., 1999; Schafermeyer et al., 1991; Schriger et al., 1991; Sheerin and de Frein, 2007; Walton et al., 1995; Waninger et al., 2001). Most evidence in the literature was based on biomechanical studies with volunteers. The studies showed that immobilization with a board and collar and head immobilization between towels or foam wedges provided the most stable biomechanical immobilization (Huerta et al., 1987; Perry et al., 1999). The addition of the board to the cervical collar provided statistically significantly more immobilization than a collar by itself (Chandler et al., 1992; Graziano et al., 1987). There were not enough studies to recommend exact types of collars. Certain forms of strapping, if applied appropriately in terms of location and tightness may further reduce lateral thoraco-lumbar spinal movement, but the clinical relevance of this reduction is not known (Mazolewski and Manix, 1994; Peery et al., 2007).

The use of rigid boards can lead to discomfort at the occiput and sacrum and increased pressures that can lead to tissue necrosis (Chan et al., 1996; Hauswald et al., 2000; Main and Lovell, 1996; Sheerin and de Frein, 2007; Walton et al., 1995). Cushioning the board can lead to increased comfort and decrease the amount of pressure at the occiput and sacrum without compromising biomechanical immobilization (Chan et al., 1996; Hauswald et al., 2000; Main and Lovell, 1996; Sheerin and de Frein, 2007; Walton et al., 1995). Several studies examined the effects of duration of immobilization on 


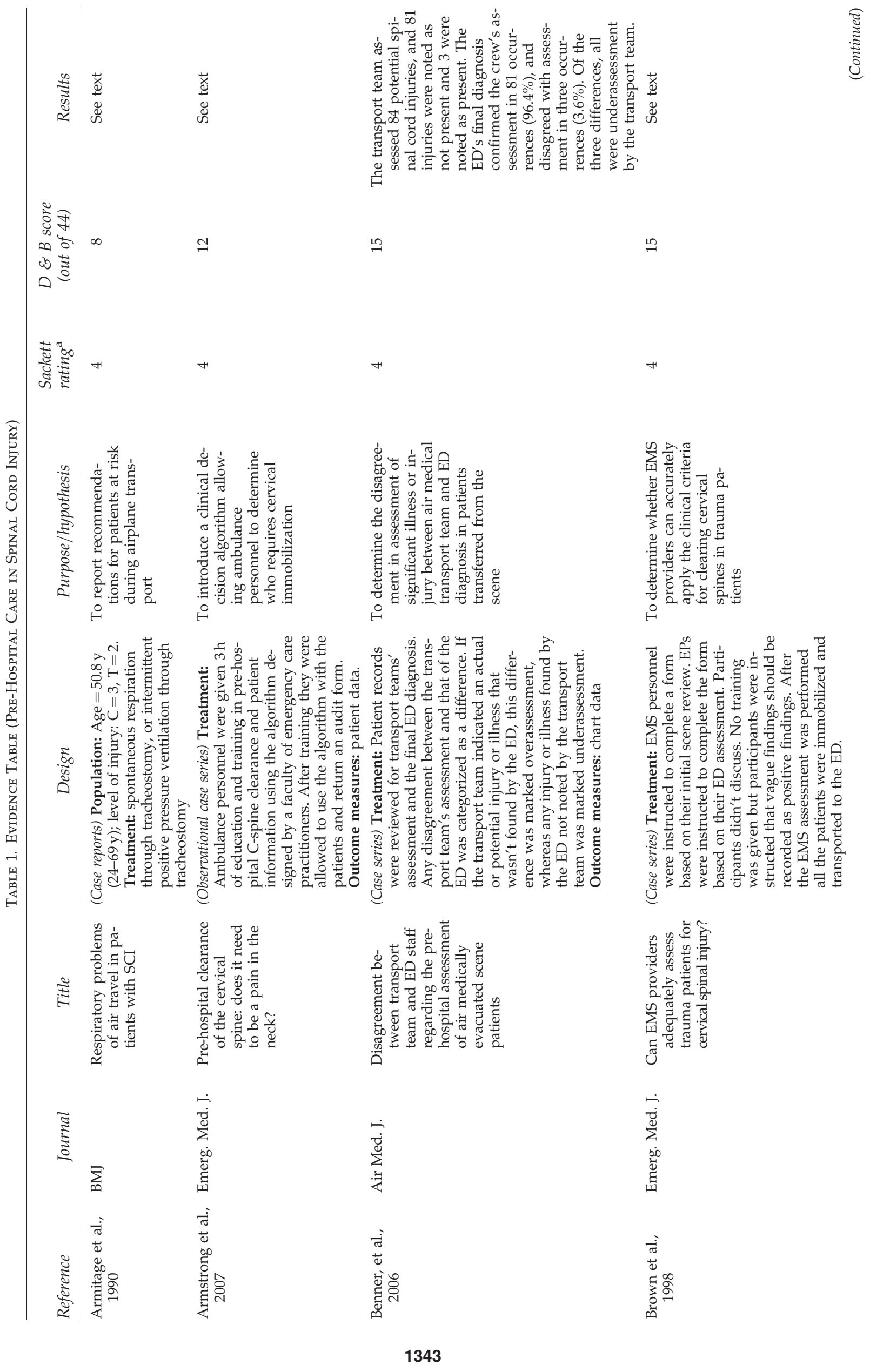




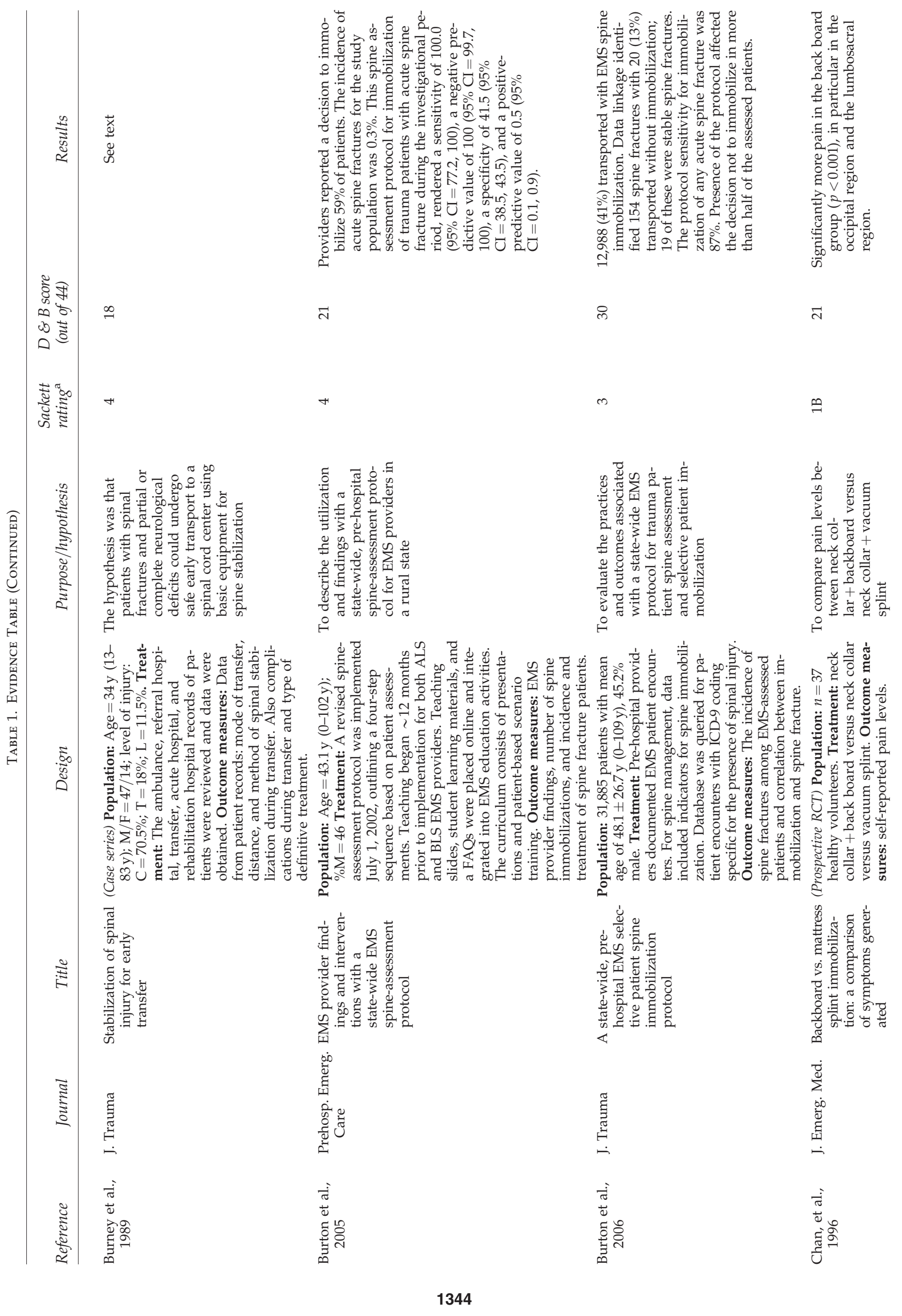




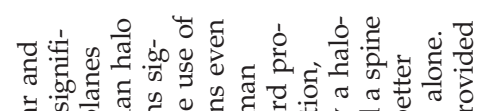

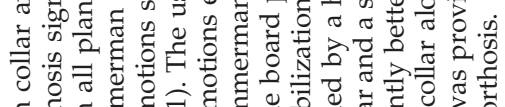

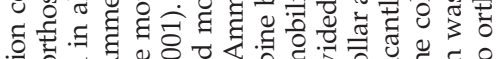

.

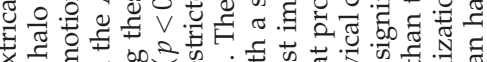

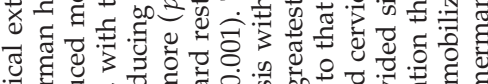

ฮै

Mํ.

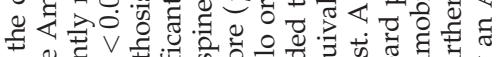

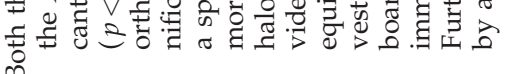

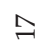

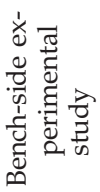

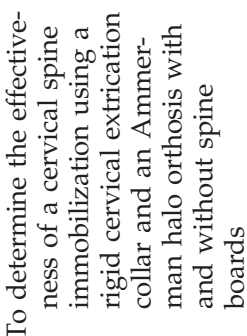

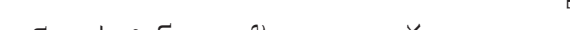

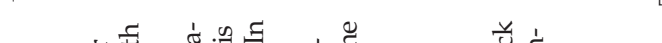

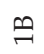

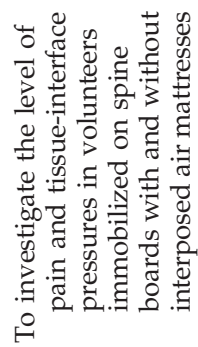

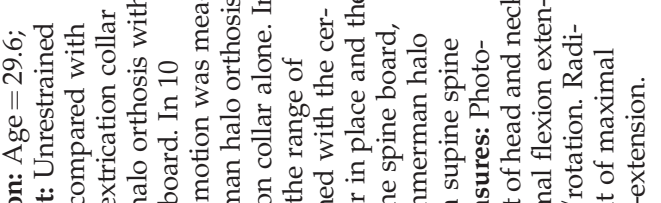

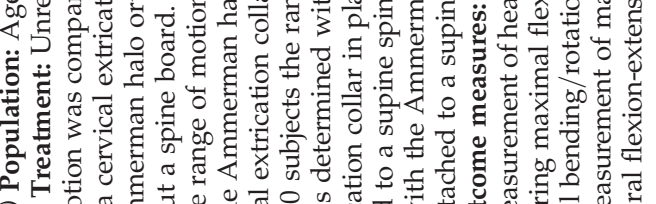

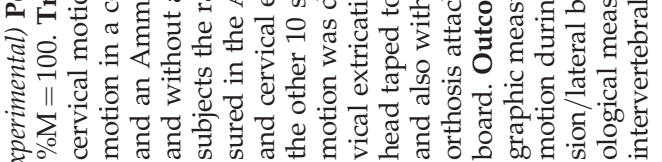
通

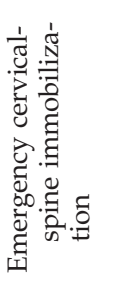

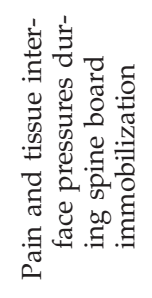

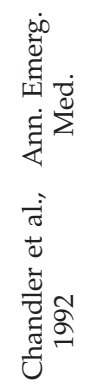

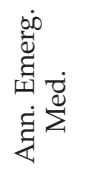

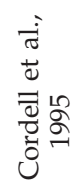




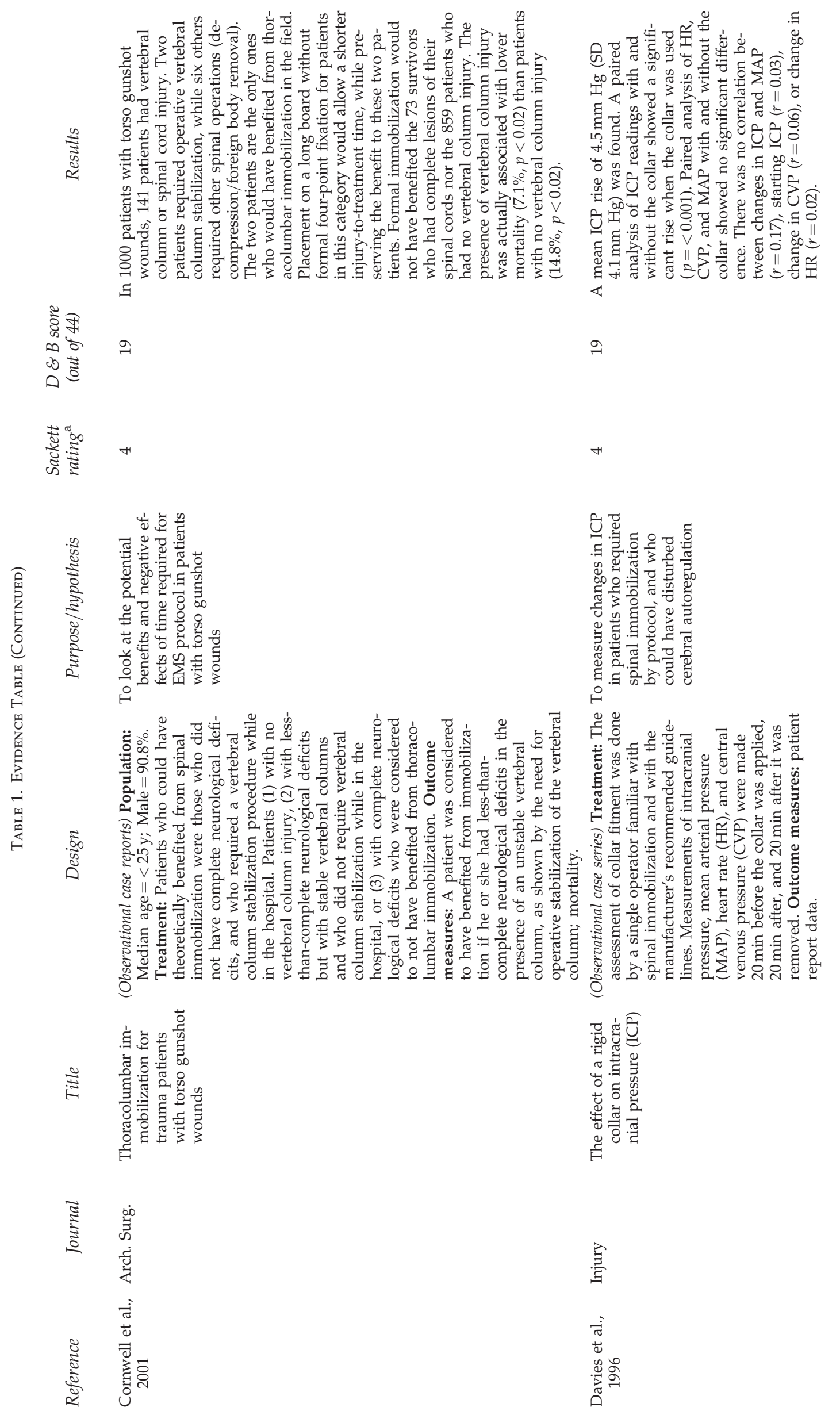




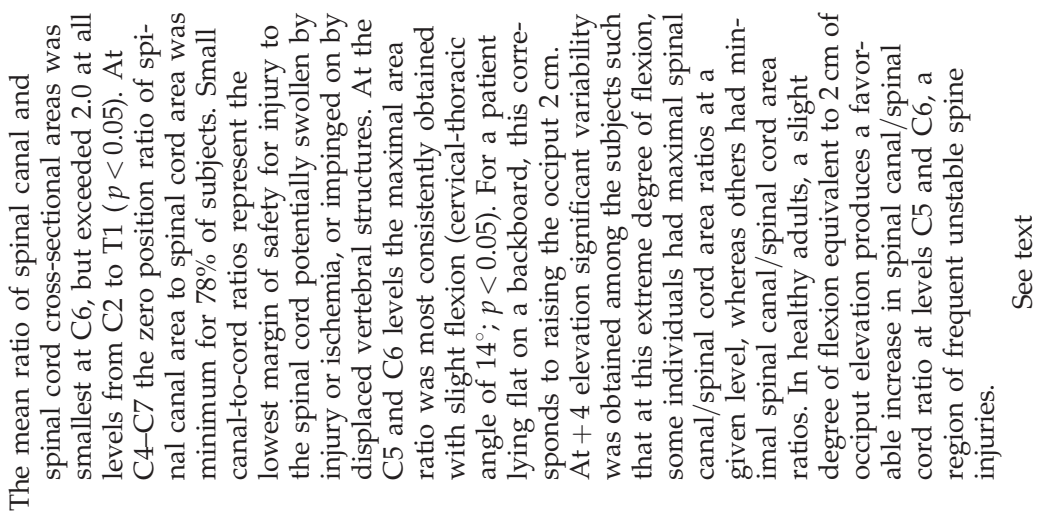

2

든

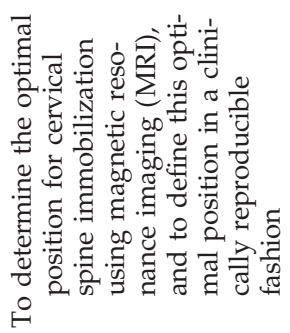

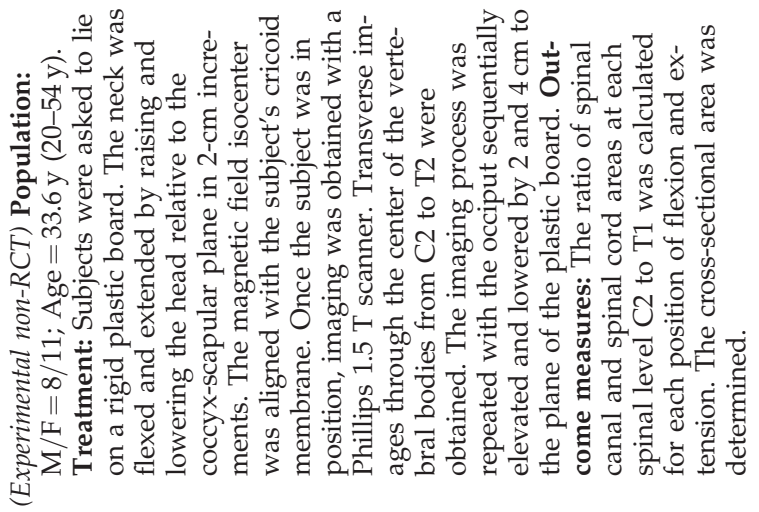
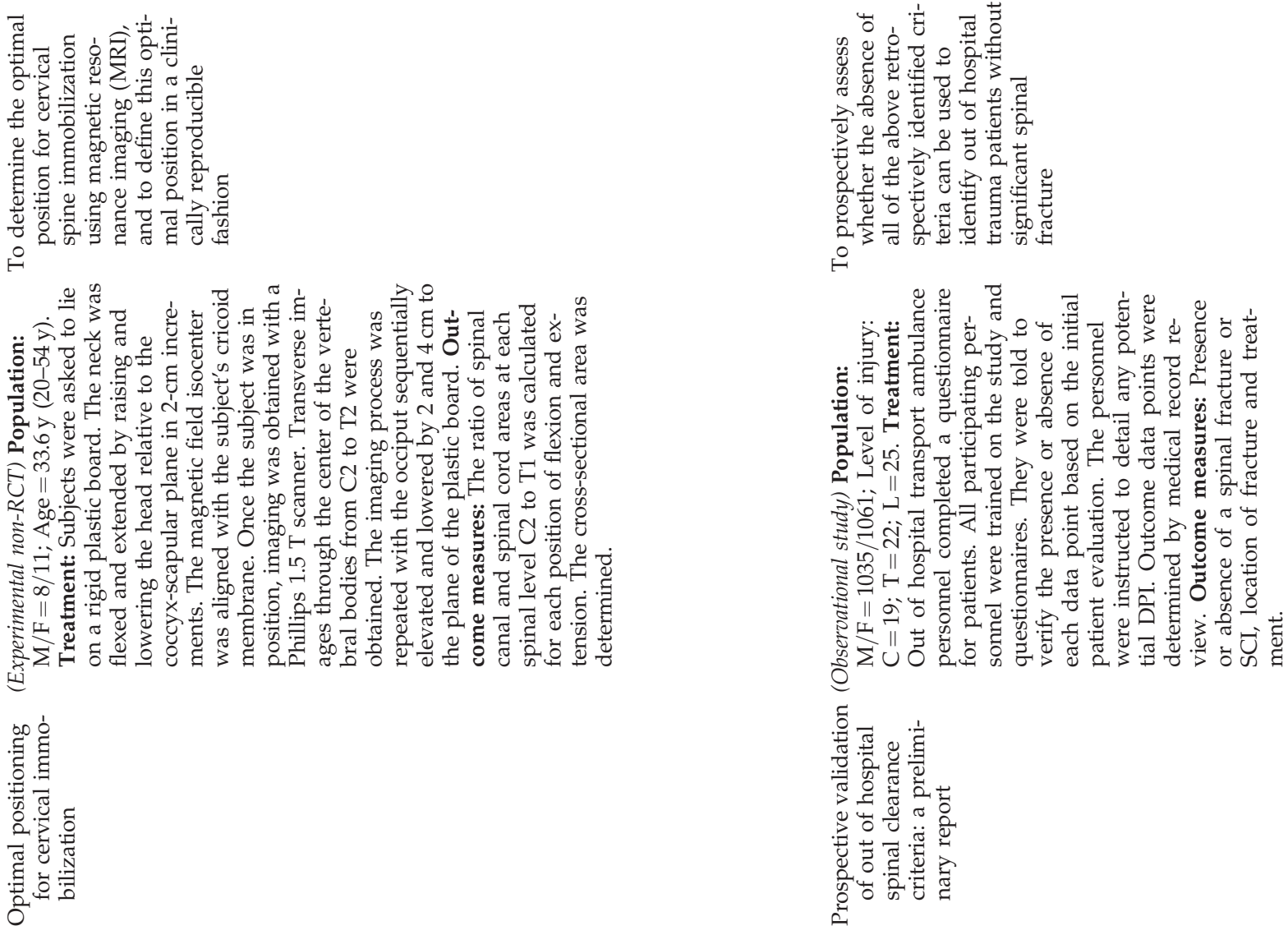

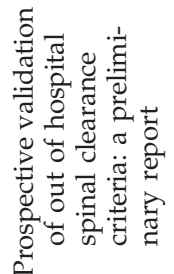

苞

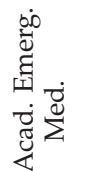

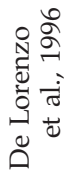

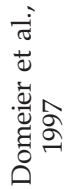




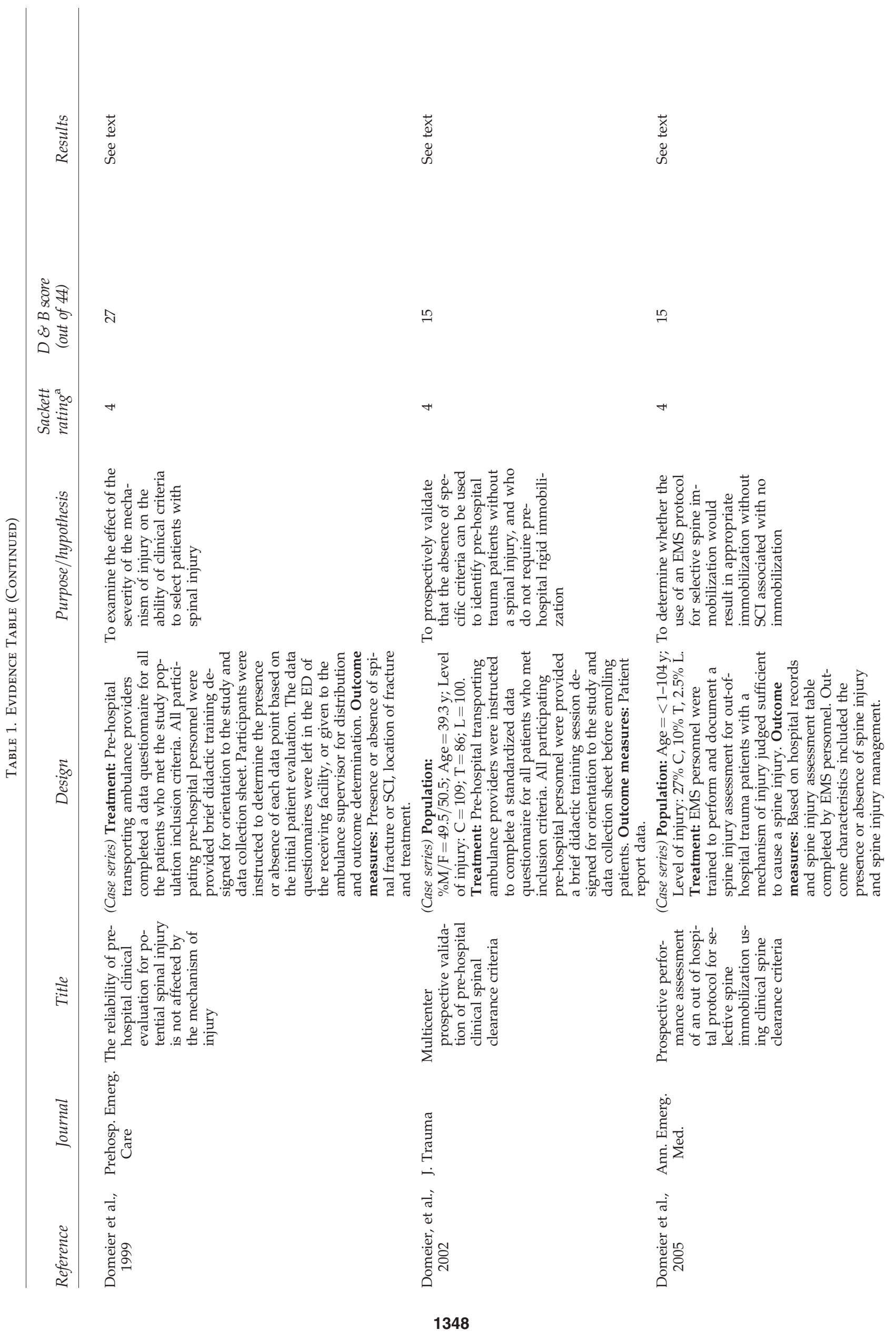




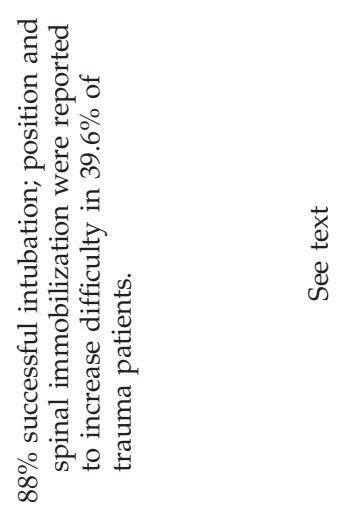

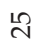

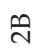

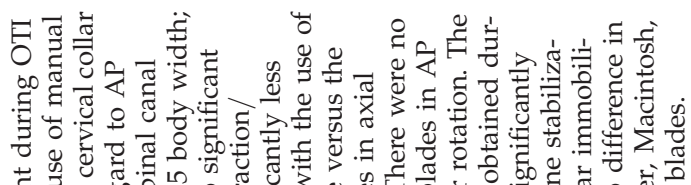
范药

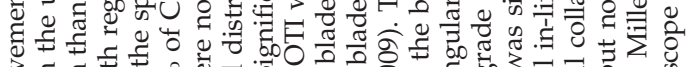

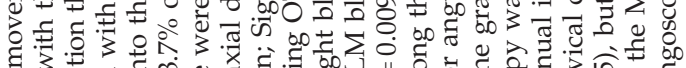

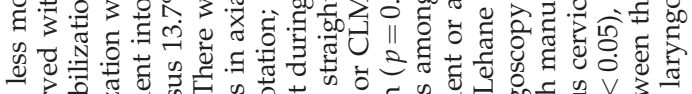

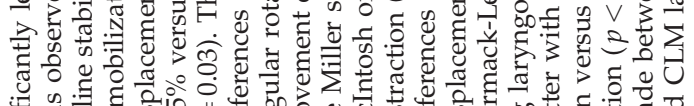

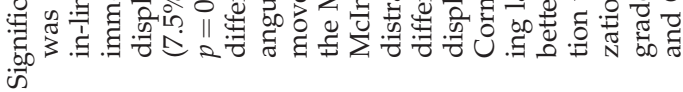

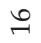

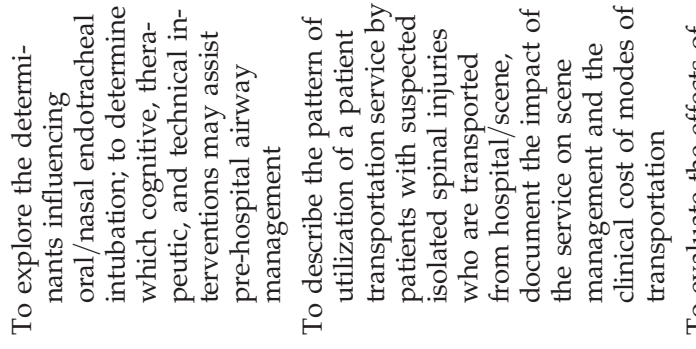

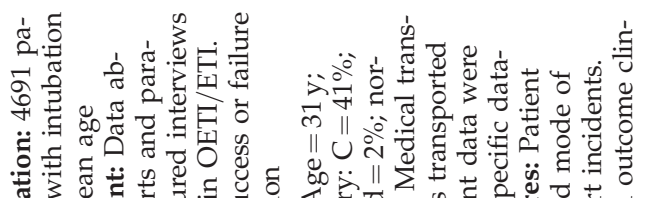

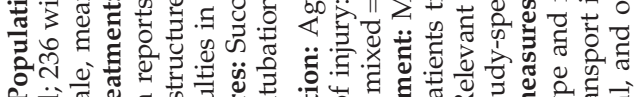

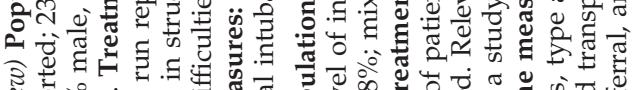

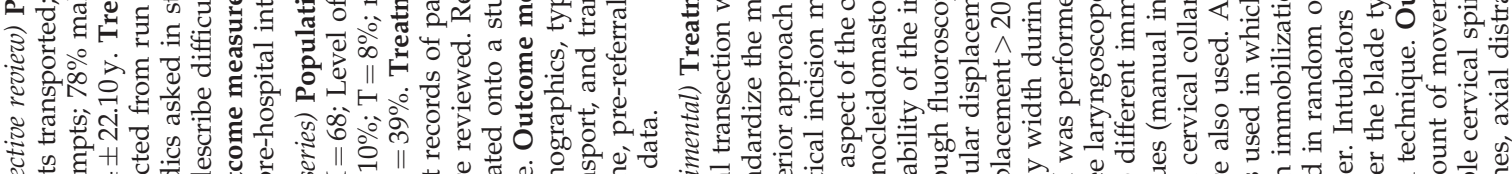

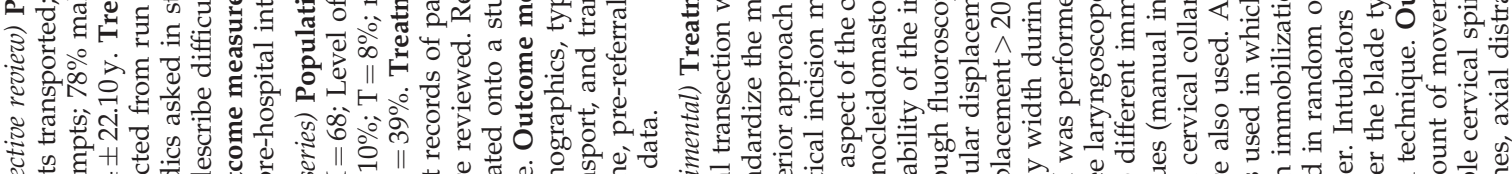

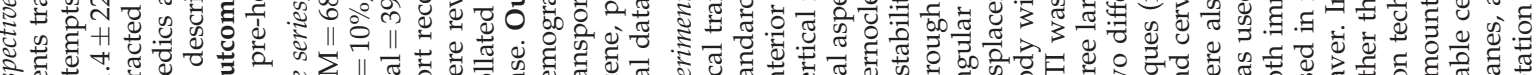

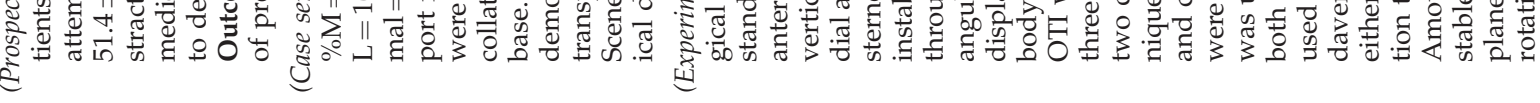

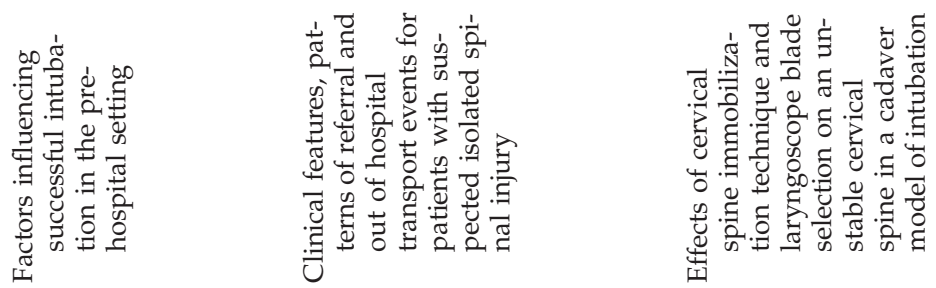

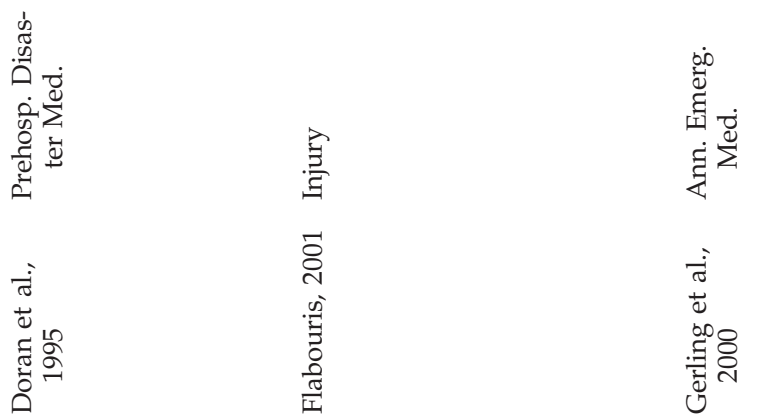




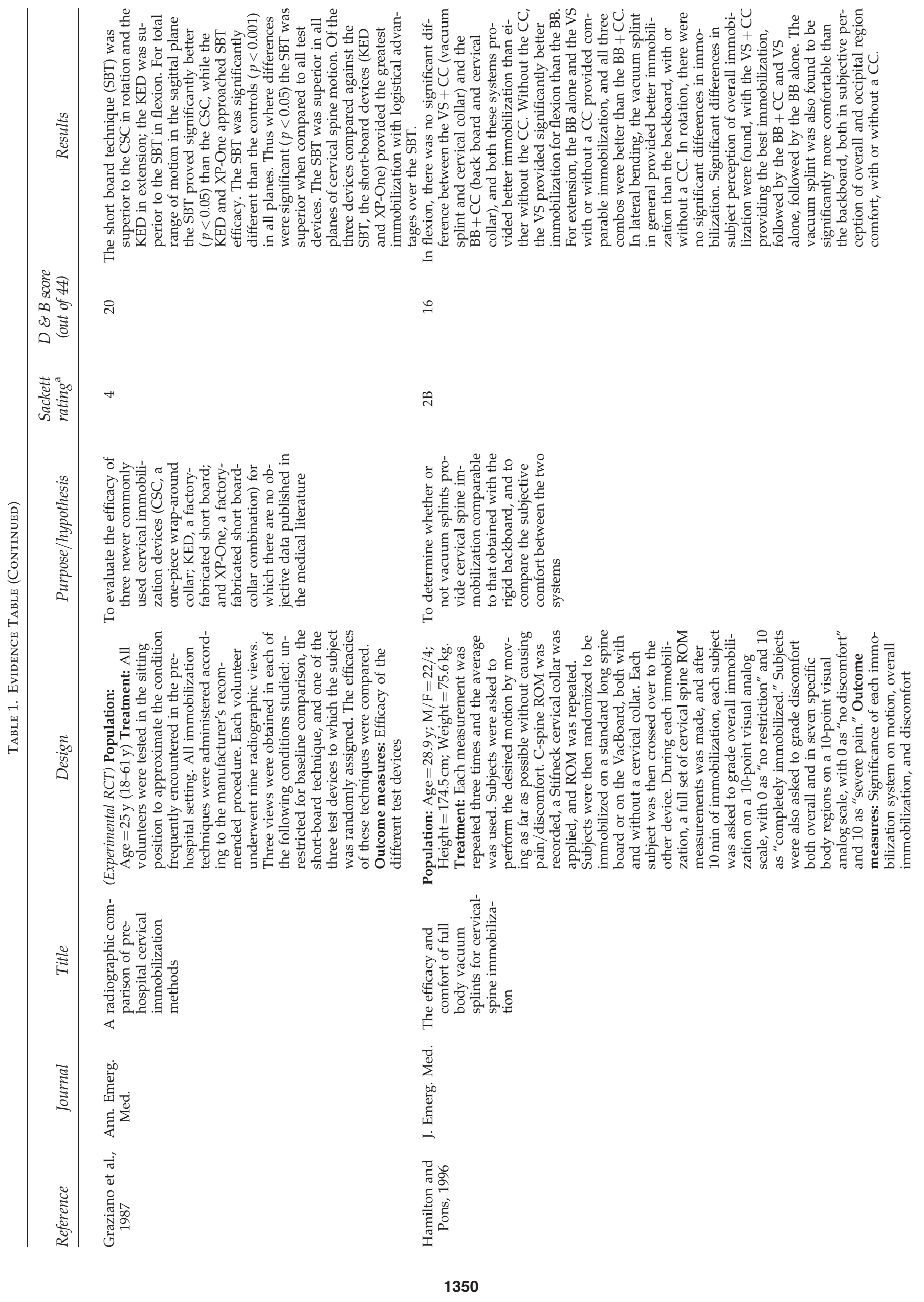




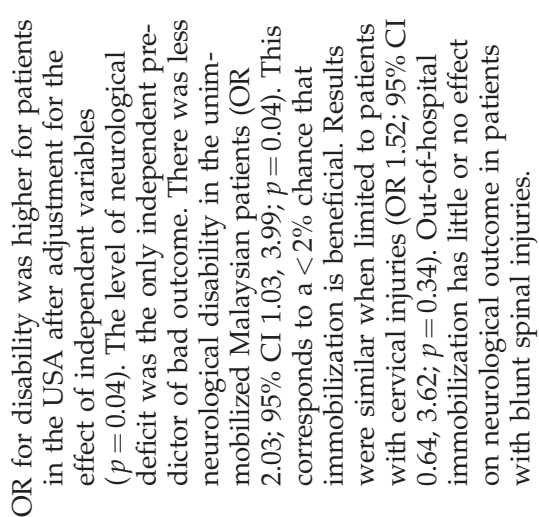

ก

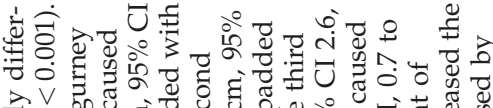

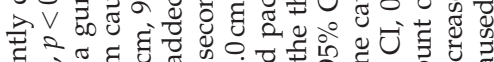

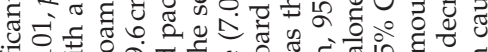

月ं

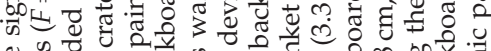

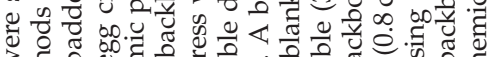

3.

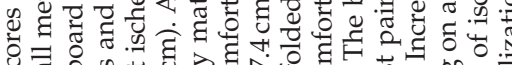

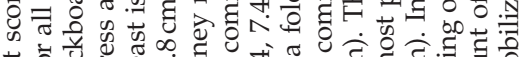

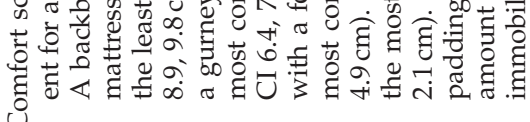

ते

สิ

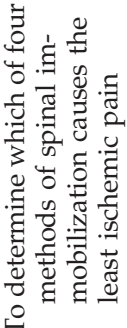

离苟

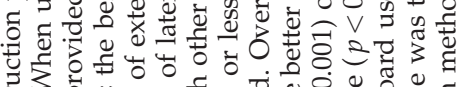
క

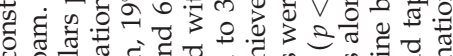

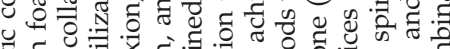
塻

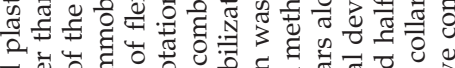

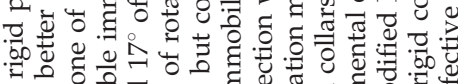
o 守

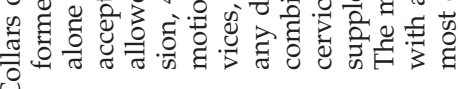

$\therefore$

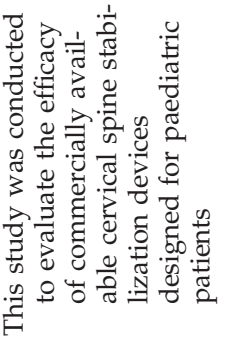

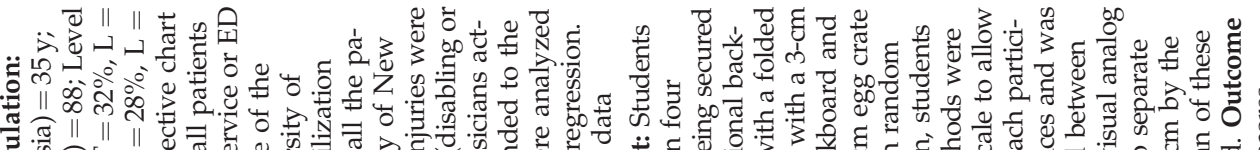

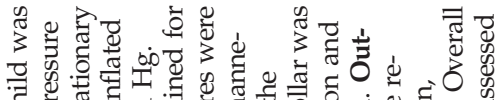
可 Iㅔ II o.

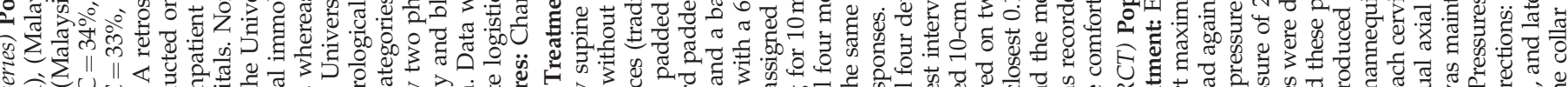

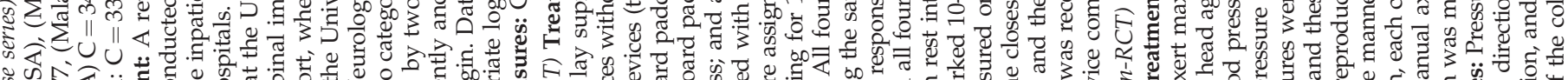

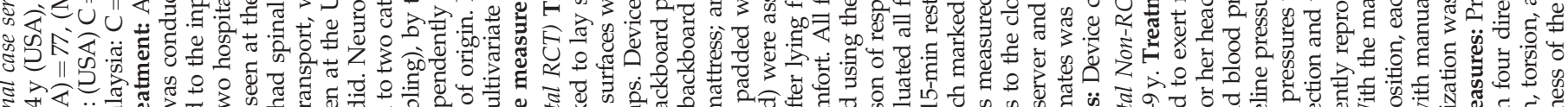

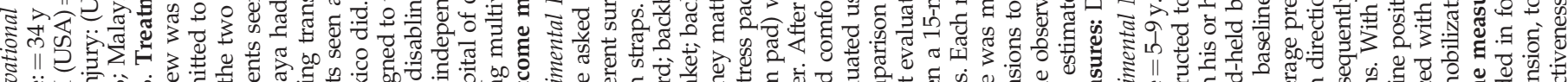

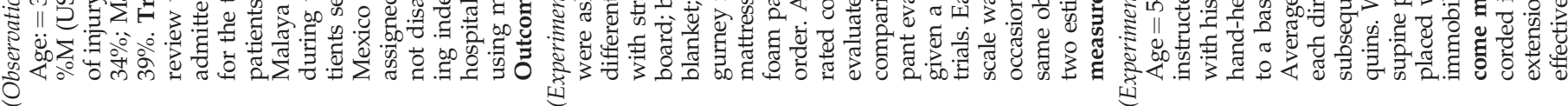

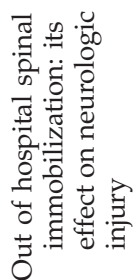

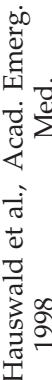

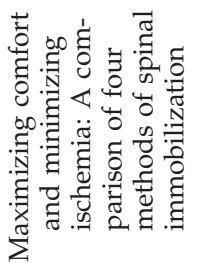

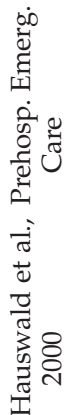

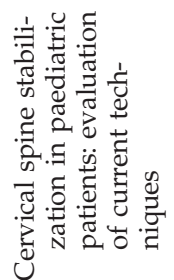

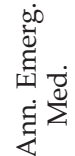

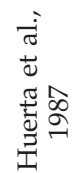




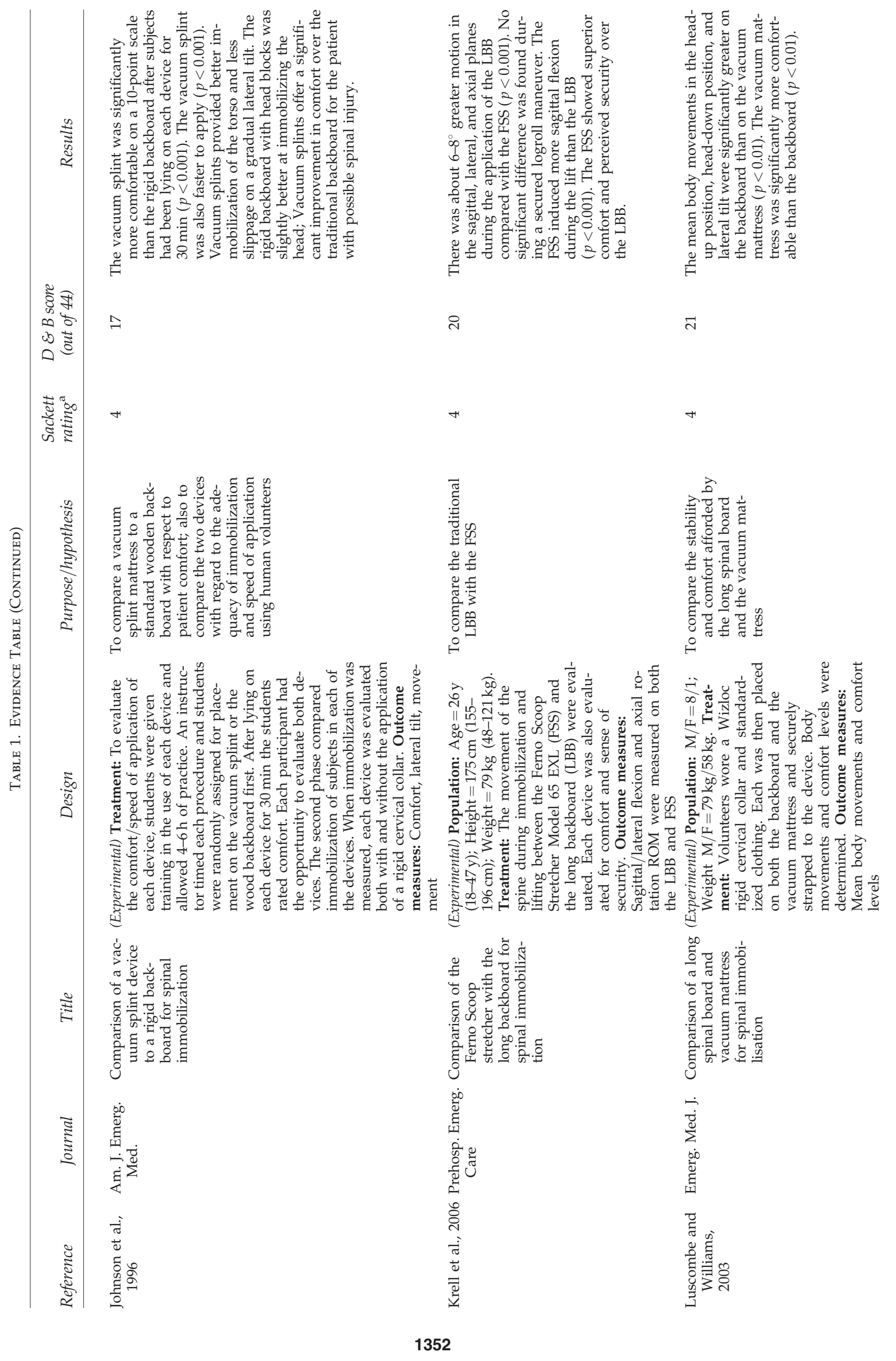




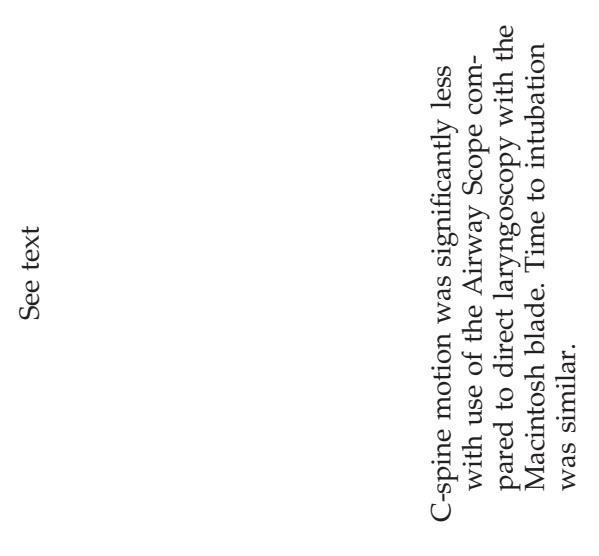

$\stackrel{12}{\sim}$

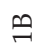

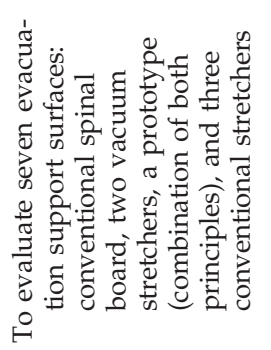

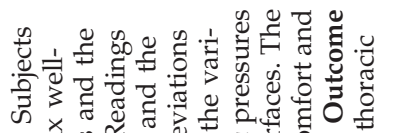

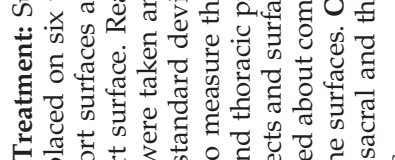

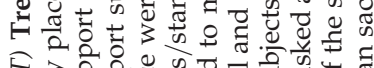

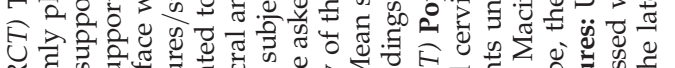

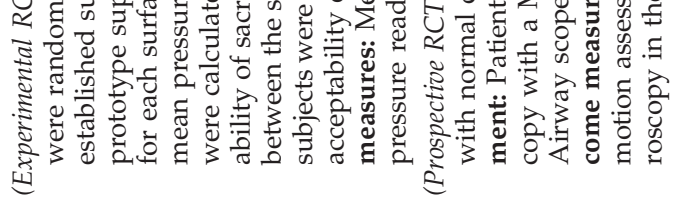
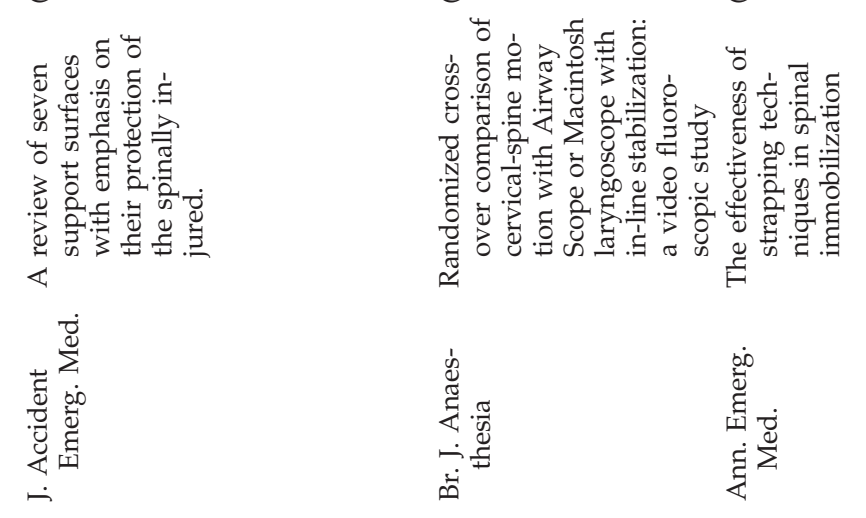

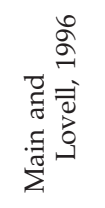

$\stackrel{2}{2}$

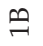

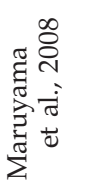

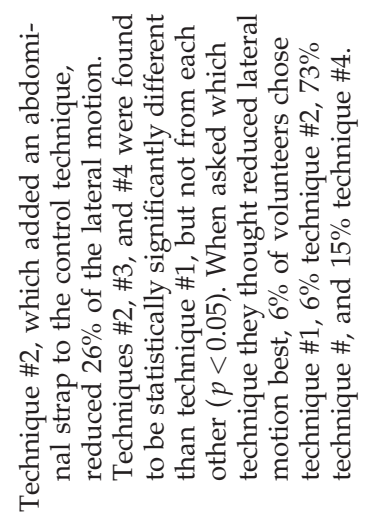

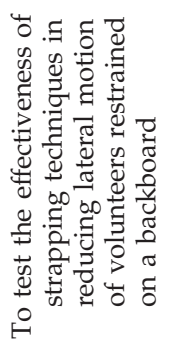

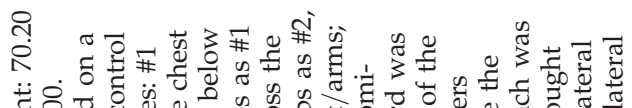

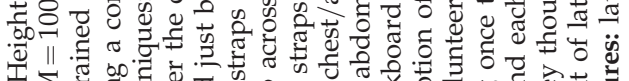

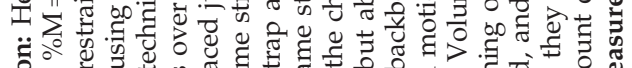
过

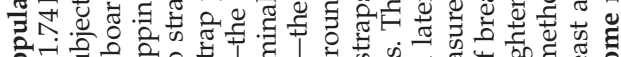

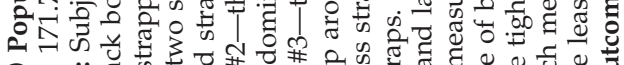

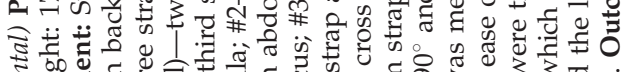

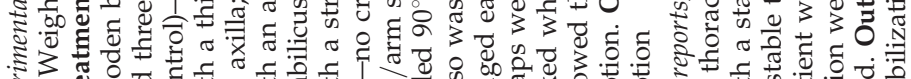

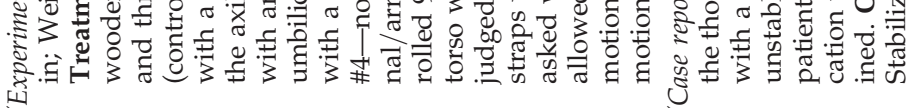
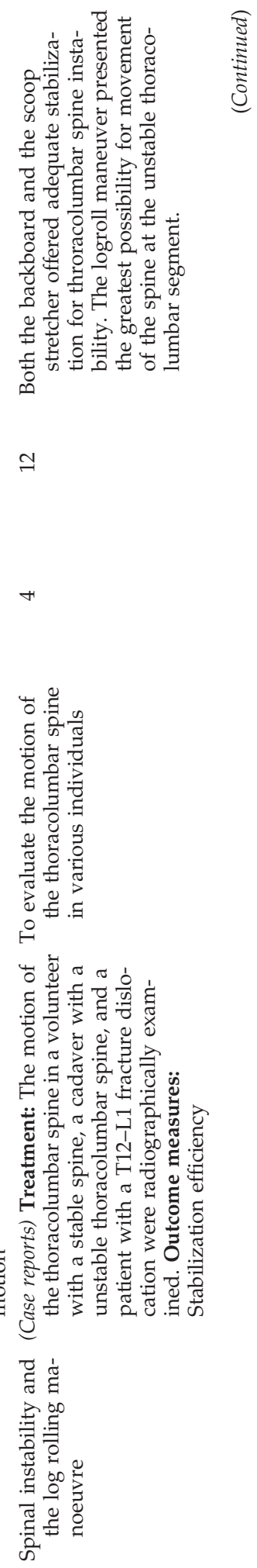

$\approx$

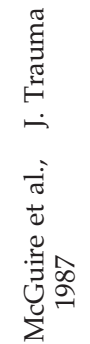




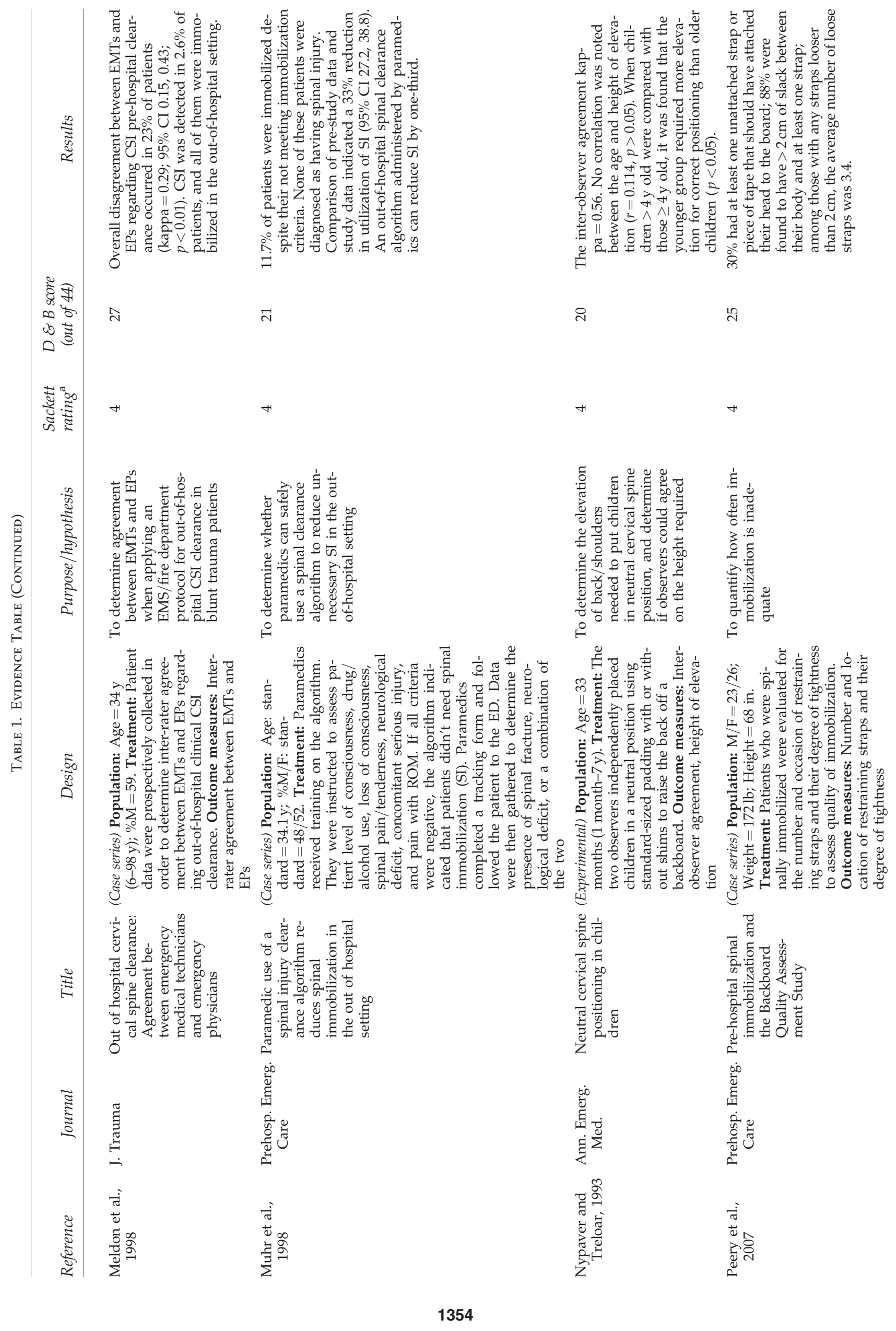




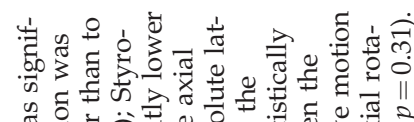

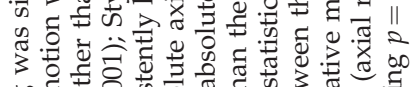

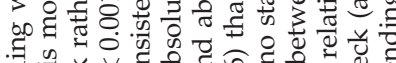

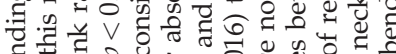

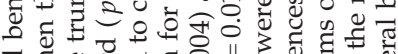

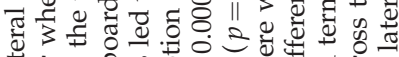

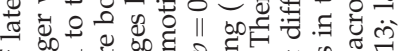

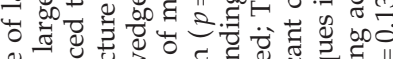
o

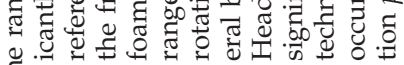
器

ก)

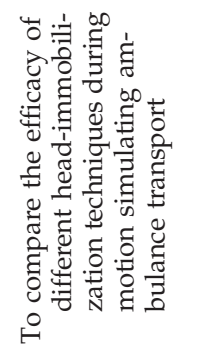

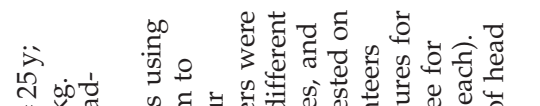

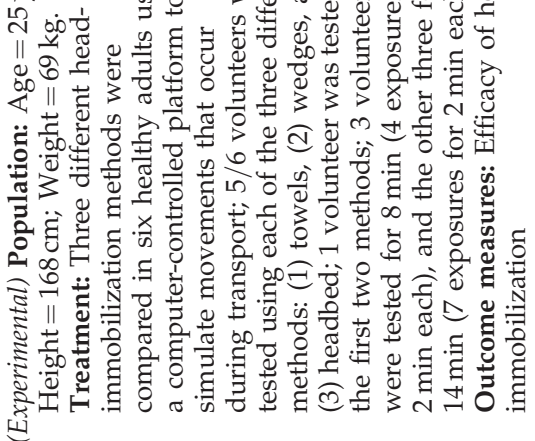

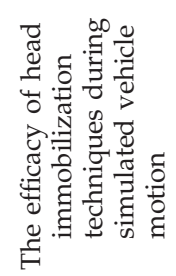

है
के
के
हें
चे
हैं

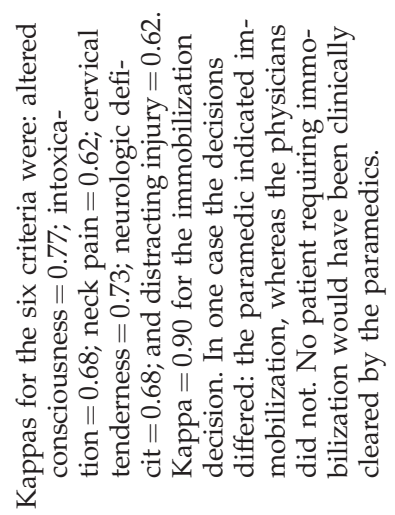

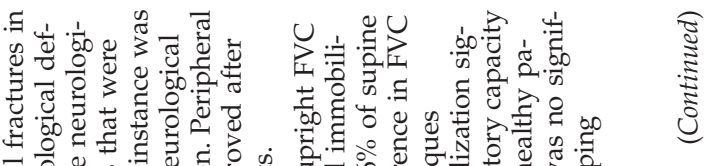

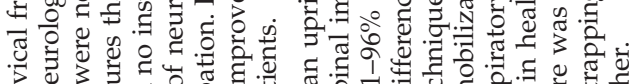

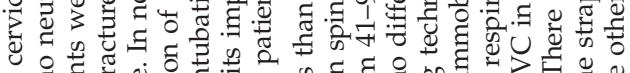

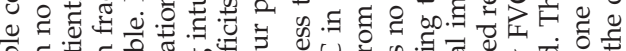

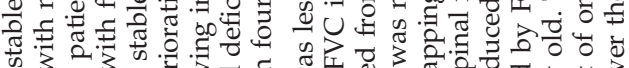
引

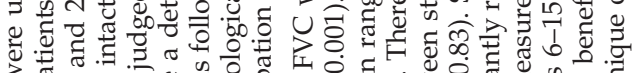
5)

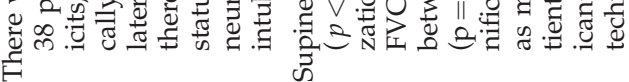

a)
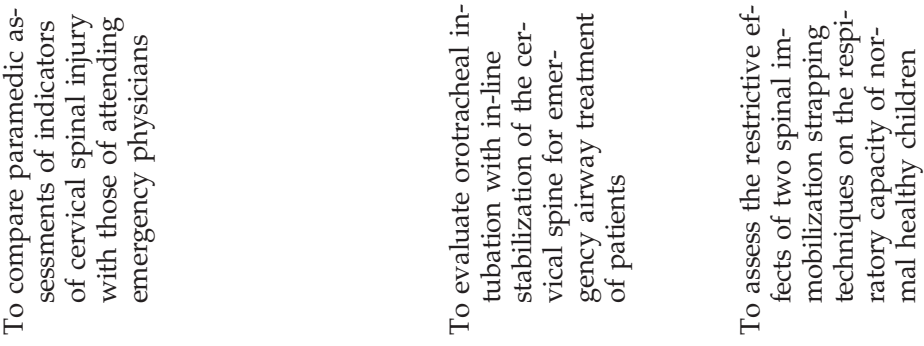

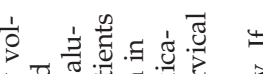

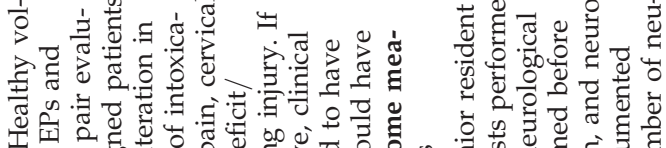

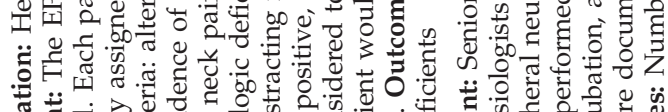

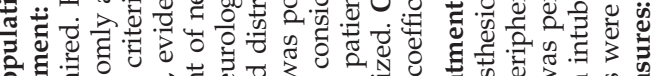

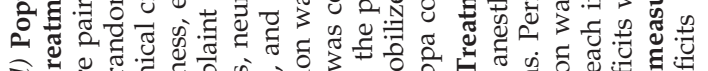

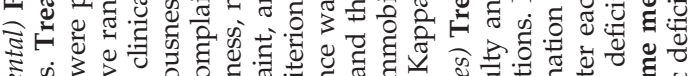

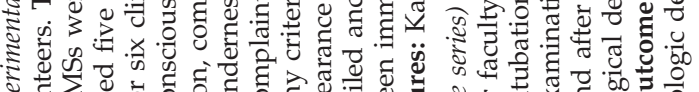

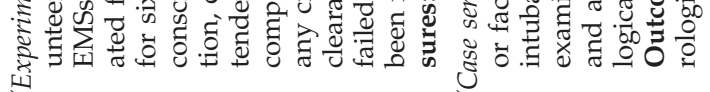

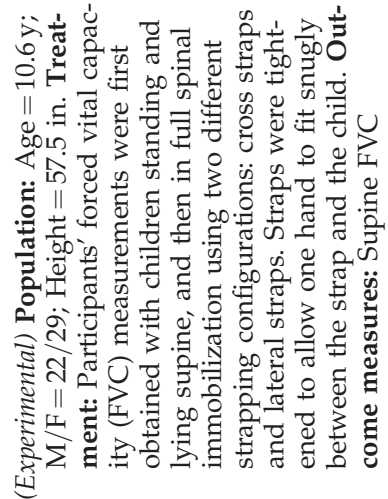
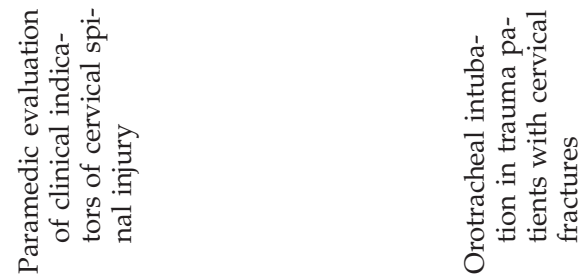

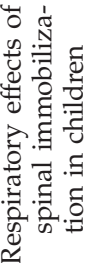

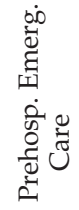

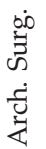

宸离

焉到
¿่

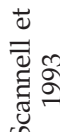




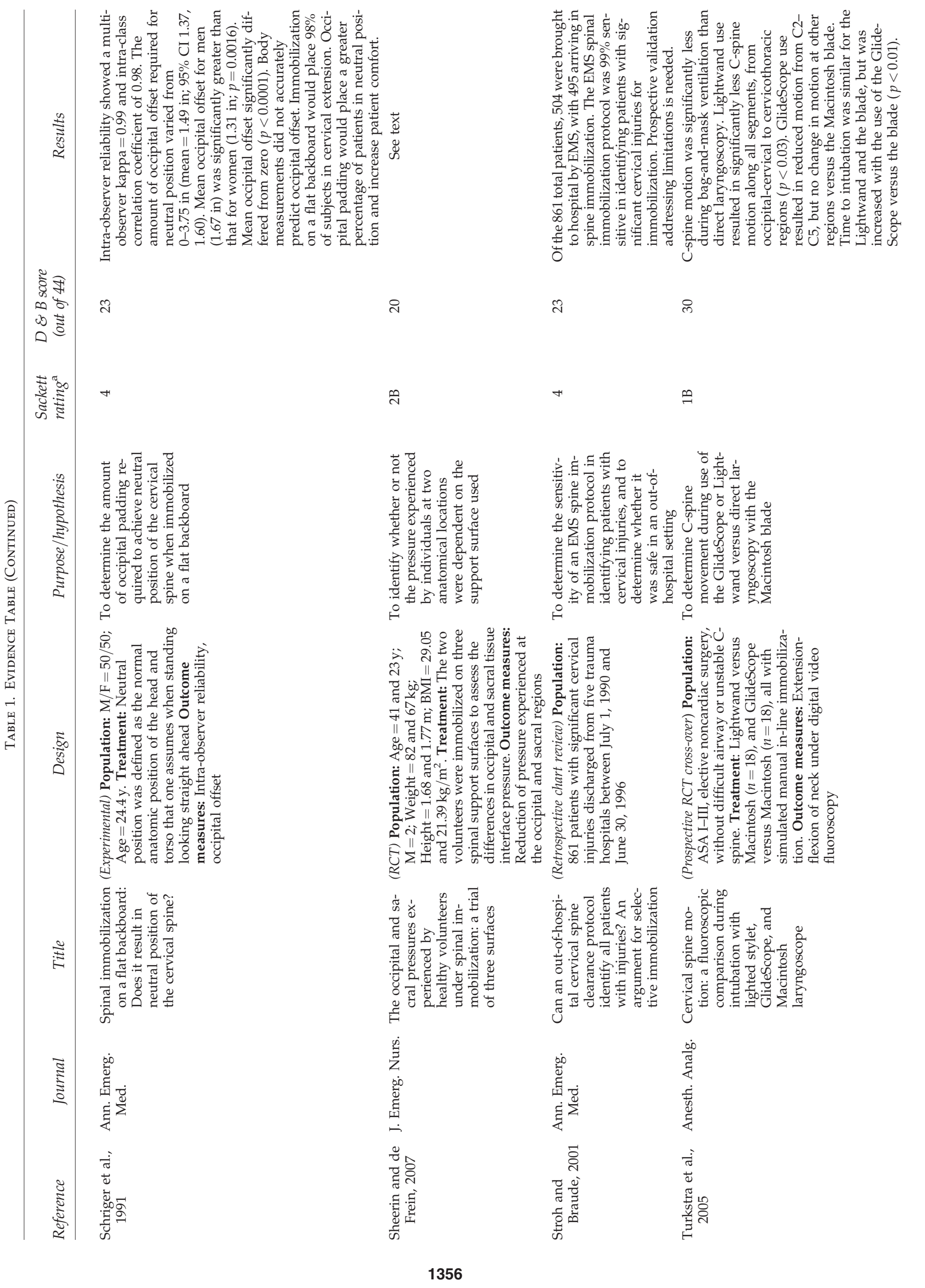




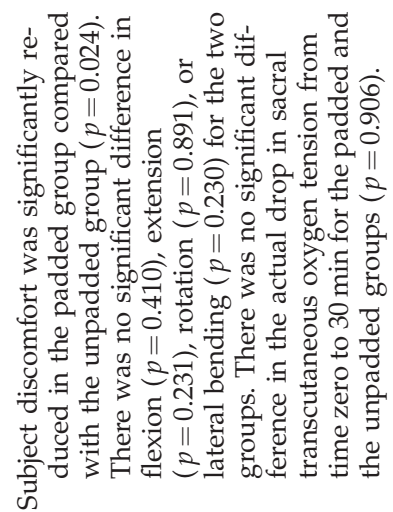

$\sqrt[2]{2}$

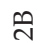

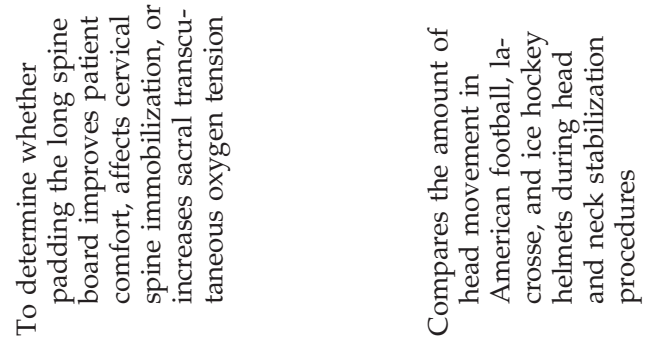

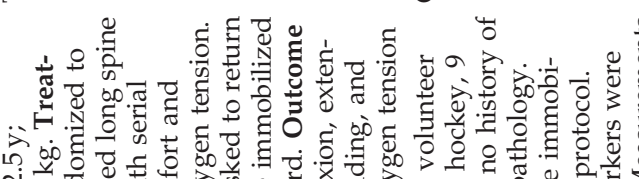

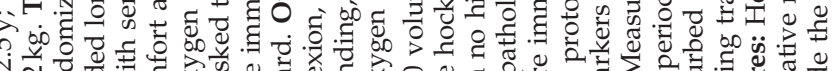

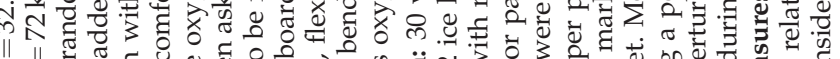

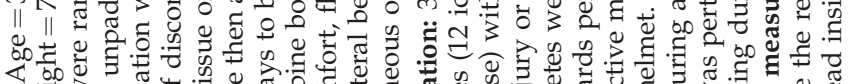

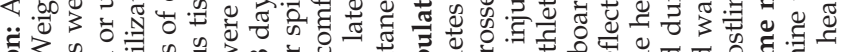

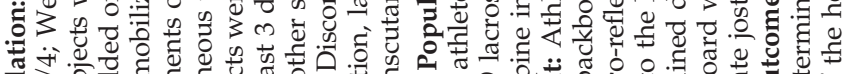

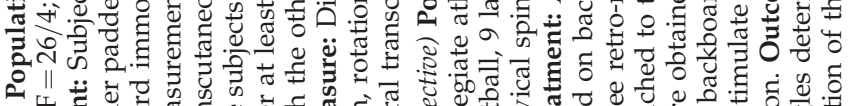

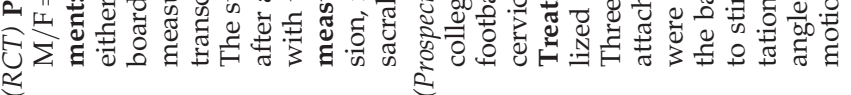
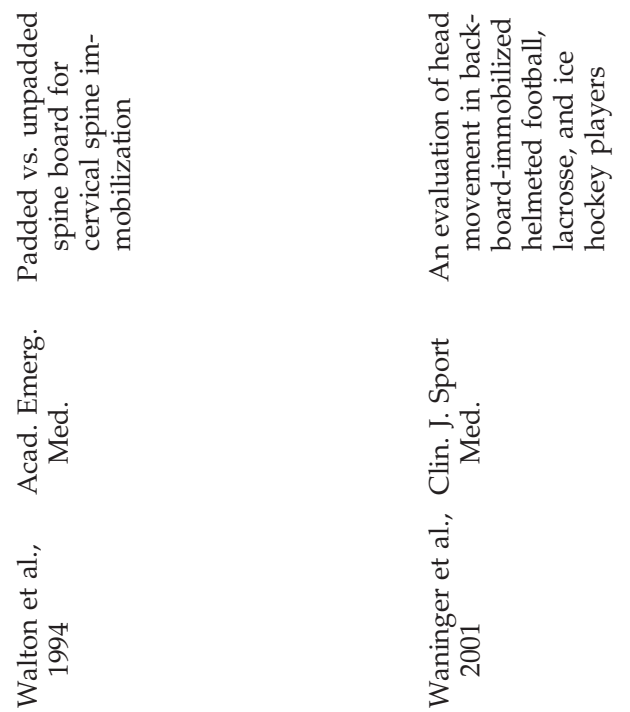

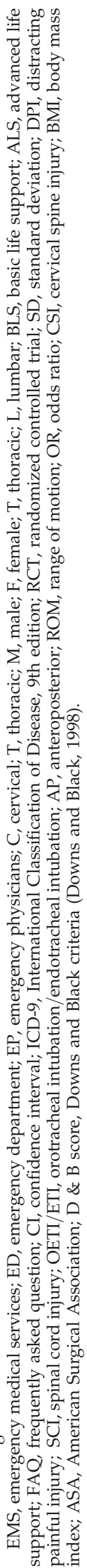


body tissue pressure (Chan et al., 1996; Hauswald et al., 2000; Main and Lovell, 1996; Sheerin and de Frein, 2007; Walton et al., 1995); however, no study evaluated what constitutes a safe duration of immobilization on a hard board, although tissue interface pressures were elevated even after short periods of rigid immobilization (Main and Lovell, 1996; Sheerin and de Frein, 2007; Walton et al., 1995). None of the studies assessed time on hard board and the clinical outcome of pressure sores. As a result, there is no firm time point cited in the literature after which immobilization should be discontinued.

Main and Lovell (1996) performed an experimental randomized controlled trial in which subjects laid on six different support surfaces and surface pressure readings were obtained both at the sacral region and the thoracic region. Results showed that the traditional spinal board had the highest sacral reading of $233.5 \mathrm{~mm} \mathrm{Hg}$ and the highest thoracic reading of $82.9 \mathrm{~mm} \mathrm{Hg}$, versus other forms of stretchers such as the York Two stretcher, for which readings of $46 \mathrm{~mm}$ $\mathrm{Hg}$ and $21 \mathrm{~mm} \mathrm{Hg}$, respectively, were obtained. The study also found that the traditional board lacked support for the lumbar lordosis. Differences in pressure readings in the various board surfaces and designs may lead to differences in the occurrence of pressure sores in the setting of prolonged transportation times and SCI (Main and Lovell, 1996).

Sheerin and de Frein (2007) conducted an experimental study examining volunteers on different support surfaces and assessed occipital and sacral tissue interface pressures. The highest pressure readings were seen with the traditional unpadded spinal board. They observed that occipital and sacral pressures were lowest with a vacuum mattress device (Sheerin and de Frein, 2007).

Mazolewski and Manix examined different techniques of strapping in spinal immobilization using an experimental study in which subjects were restrained on a spine board. Four different techniques were evaluated and lateral spine motion was measured as the backboard was rolled $90^{\circ}$ from side to side. Motion was most reduced by placing two straps that cross over at the chest, with a third strap placed across the umbilicus (Mazolewski and Manix, 1994).

Krell and colleagues compared the scoop stretcher to the long backboard for spinal immobilization in terms of motion and comfort. There were $6-8^{\circ}$ more of sagittal motion during the application of the long backboard compared to the scoop board. The scoop board was also perceived to be more comfortable compared to the traditional board (Krell et al., 2006).

There are few published studies evaluating spinal immobilization for children. Only two of the identified publications studied pediatric spinal immobilization (Nypaver and Treloar, 1994; Schafermeyer et al., 1991). There are anatomic differences between adults and children that may prevent valid generalizations of the adult literature to spinal immobilization in a pediatric population. These include increased head:body size ratio, as well as a more posterior occiput relative to the spinal plumb line in children than in adults. With the relative paucity of literature evaluating pediatric immobilization, and the concerns regarding generalizability of adult findings to this population, the group decided to restrict recommendations made in this review to individuals over the age of 12 years.
Question 2. During airway manipulation in the prehospital setting, what is the ideal method of spinal immobilization?

Most studies examining airway management were performed in the hospital rather than in the pre-hospital setting (Doran et al., 1995; Maruyama et al., 2008; Scannell et al., 1993; Turkstra et al., 2005). Intubations in the studies were done by senior anesthesia residents or fully-trained anesthesiologists. The studies did not assess intubations done by emergency medical technicians in the field, and there was only one article examining pre-hospital intubation and factors influencing successful intubation in the pre-hospital setting. This prospective study evaluated 4691 transported patients, of which 236 required intubation. The intubation success rate was $88 \%$, and was considered to be technically more challenging, especially in the setting of spinal immobilization (Doran et al., 1995).

The available clinical studies evaluating the impact of airway control using in-line cervical stabilization did not find worsening of neurologic status after airway management (Maruyama et al., 2008; Scannell et al., 1993). Anatomical studies of in-line stabilization with a Miller blade showed less cervical movement compared to use of a cervical collar alone (Gerling et al., 2000).

Several randomized cross-over trials were performed assessing newer forms of indirect intubation in patients undergoing general anesthesia for elective surgeries with live fluoroscopy (Maruyama et al., 2008; Turkstra et al., 2005). Indirect methods of intubation such as the Lightwand with manual in-line immobilization were found to cause less cervical motion than direct laryngoscopy with a Miller blade and in-line immobilization (Maruyama et al., 2008; Turkstra et al., 2005).

Question 3. What is the impact of pre-hospital transport time to definitive care on the outcomes of patients with acute SCI?

There is little evidence regarding the impact of pre-hospital transport time to definitive care on the outcomes of patients with acute SCI. However, patients transported within $24 \mathrm{~h}$ for treatment fared better than those transported after $24 \mathrm{~h}$. It is unknown if there were confounding factors in those patients transported after $24 \mathrm{~h}$ that negatively affected outcomes, such as medical comorbidities or concurrent injuries that hindered earlier transportation, and thus negatively impacted the results of spinal cord injury treatment. Air and ground transport are both safe when spinal precautions are taken for transport (Armitage et al., 1990; Burney et al., 1989; Flabouris, 2001), and these studies did not document the development of any ascending neurological deficits with transport (Armitage et al., 1990; Burney et al., 1989; Flabouris, 2001).

Burney and associates reviewed patients with spinal column fractures with SCI (complete or incomplete) to assess whether these patients could undergo safe early transport to an SCI center using basic equipment for spine stabilization. Transportation was achieved both by ground ambulance $(41 \%)$, helicopter $(54 \%)$, and fixed-wing aircraft $(5 \%)$, and $84 \%$ were transferred within $24 \mathrm{~h}$ of injury. No patients suffered ascending injury levels as a result of transfer. There was no significant difference found in the probability of improvement between ground and air transportation (Burney et al., 1989). 
Flabouris and colleagues reviewed the pattern of utilization of a medically-staffed transportation service by patients with suspected spinal injuries from a hospital or from a scene of an accident and documented the impact of different modes of transportation. The majority (93\%) of transfers were by helicopter, followed by fixed-wing aircraft $(3.5 \%)$, and lastly road vehicles. Mean duration of inter-hospital transport ( $42 \pm 28 \mathrm{~min}$ ) was longer than from scene transfers $(19 \pm 12 \mathrm{~min})$, but this was similar to road vehicle transfers $(45 \pm 26 \mathrm{~min})$. Rural hospitals were the referral source for $55 \%$ of inter-hospital transports. The reason for $18 \%$ of all interhospital transfers was to exclude a spinal injury that could not otherwise be excluded at the referral hospital. No worse neurological outcomes occurred as a result of transportation (Flabouris, 2001).

Question 4. What is the role for pre-hospital care providers in cervical spine clearance and immobilization?

Clinical evidence reveals that pre-hospital emergency medical technicians can be trained to apply criteria to clear patients of cervical spinal injuries and immobilize patients suspected of having a cervical spinal injury to a level similar to that of emergency physicians (Armstrong et al., 2007; Benner et al., 2006; Brown et al., 1998; Burton et al., 2005, 2006; Campbell, 1987; Domeier et al., 1997, 1999, 2002, 2005; Meldon et al., 1998; Muhr et al., 1999; Sahni et al., 1997; Stroh and Braude, 2001). There was no universal tool or triage index used in the studies.

In some series up to $8 \%$ of vertebral column injuries were not immobilized (Armstrong et al., 2007; Brown et al., 1998; Domeier et al., 2002, 2005; Stroh and Braude, 2001). However, there were no clinical consequences of not immobilizing, and none of these column injuries had associated neurological deficits (Armstrong et al., 2007; Brown et al., 1998; Domeier et al., 2002, 2005; Stroh and Braude, 2001).

Armstrong and associates examined whether the incidence of unnecessary cervical spine immobilization by ambulance personnel could be safely reduced through the implementation of an evidence-based algorithm. Following a training program, paramedics collected data on 103 patients with potential cervical spine injuries, of which 69 (67\%) had their cervical spines cleared at the accident scene. Of these, 60 (87\%) were discharged at the scene with no clinical adverse events reported, and $9(13 \%)$ were taken to the local emergency department with minor injuries, and all were discharged home the same day. However, 34 (33\%) patients could not have their cervical spines safely cleared at the scene according to the algorithm. Of these, $4(12 \%)$ patients self-discharged themselves at the scene, and $30(88 \%)$ were conveyed to an emergency department per their procedure protocol (Armstrong et al., 2007).

Brown and co-workers examined whether emergency medical services (EMS) providers can accurately apply clinical criteria for clearing the cervical spine in trauma patients. Both emergency physicians and EMS providers indicated immobilization in $60 \%$ of patients, and their assessments differed for $21.3 \%$ of patients. Overall agreement indicated a kappa value of 0.48 , which reflects moderate agreement. The EMS providers were generally more conservative than the emergency physicians (Brown et al., 1998).

Domeier and colleagues first examined prospectively whether or not retrospectively identified criteria could be used to identify patients without significant spinal fracture outside of the hospital. By utilizing their criteria, 100\% of all cervical injuries were identified. They identified $90 \%$ of patients with thoracic injuries, and $96 \%$ of patients with lumbar injuries. There were three false-negatives, of which two had stable thoracic compression injuries, and one had a lumbar transverse fracture. Only one of these false-negatives was admitted to hospital for pain control, and was discharged within 2 days, and the remaining false-negative injuries were discharged from the emergency department. No significant spinal fractures were missed (Domeier et al., 1997).

In another study, Domeier and associates examined the reliability of a pre-hospital clinical evaluation tool in patients with different severities and mechanisms of injury. In all, 1059 patients were assessed in the high-risk group, of which $9.4 \%$ had injuries, and 5423 low-risk group patients were assessed, of which $2 \%$ had injuries. The criteria identified $97 \%$ of injuries in the high-risk group, and $94 \%$ in the low-risk group. The mechanism of injury did not affect the ability of the clinical criteria to predict spinal injury (Domeier et al., 1999).

In 2005, Domeier and colleagues assessed whether the protocols developed allowed EMS providers to appropriately immobilize patients with spinal injuries. The sensitivity of the EMS protocol was $92 \%(95 \%$ CI $89.4,94.6)$, and $8 \%$ of patients with spinal injuries did not have immobilization; however, none of the non-immobilized patients sustained cord injuries. EMS providers also immobilized $12 \%$ of patients not required by the protocol. The use of the selective immobilization protocol resulted in spine immobilization for most patients with spinal injury, without causing harm in cases in whom immobilization was withheld (Domeier et al., 2005).

Muhr and associates also examined training paramedics to use a clearance algorithm. If patients met all the criteria, paramedics could transport them without spine immobilization. They found that there was a 33\% reduction in the utilization of spinal immobilization compared to pre-study data (Muhr et al., 1999).

Further research is needed to determine a universal triage system that can be implemented to train emergency medical technicians in the pre-hospital setting. However, the results of the systematic review for this question must be balanced with the realities of geographic variations in law and health policy, and the varying risk tolerance of EMS systems in different regions.

\section{Recommendations}

All recommendations were derived from the systematic reviews, statements from the authors, and the Delphi process. The latter was reported using the level of agreement and the comments and suggestions of experts.

Question 1. What is the optimal type and duration of prehospital spinal immobilization in patients with acute SCI?

- Immobilization of patients with SCI during the prehospital setting should include a cervical collar, head immobilization, and a spinal board.

- Patients should be transferred off the hardboard on admission to a facility as soon as is feasible to minimize time on the hardboard. If patients are awaiting transfer to another institution, they should be taken off the hardboard while awaiting transfer. 
- Padded boards or inflatable bean bag boards should be utilized to reduce pressure on the occiput and sacrum.

- These recommendations are intended for adults and children over the age of 12 years.

Question 2. During airway manipulation in the prehospital setting, what is the ideal method of spinal immobilization?

- Airway management of acute SCI patients requiring intubation in the pre-hospital setting should include the use of manual in-line cervical spine traction.

- Intubation of patients with acute SCI in the pre-hospital setting should not rely solely on cervical collar neck immobilization.

- Indirect methods of intubation may cause less cervical movement than with direct laryngoscopy with a Miller blade.

Question 3. What is the impact of pre-hospital transport time to definitive care on the outcomes of patients with acute SCI?

- Transport of patients with acute traumatic SCI to the definitive hospital center for care should occur within $24 \mathrm{~h}$ of injury.

Question 4. What is the role for pre-hospital care providers in cervical spine clearance and immobilization?

- Emergency medical personnel in the pre-hospital setting can be trained to apply criteria to clear patients of cervical spinal injuries and immobilize patients suspected of having a cervical spinal injury.

- The implementation of this recommendation will likely be impacted by regional variations in law and health policy.

There is insufficient evidence to make recommendations for children, and the authors have concerns regarding the applicability of adult recommendations to pediatric patients.

\section{Summary}

Pre-hospital care of patients with potential spinal cord injury requires great care to minimize secondary SCI and potential morbidity related to spinal immobilization. This systematic review provides an evidentiary table and a summary of the review, in addition to recommended guidelines based on use of the Delphi method by a group of experts.

\section{References}

Armitage, J.M., Pyne, A., Williams, S.J., and Frankel, H. (1990). Respiratory problems of air travel in patients with spinal cord injuries. BMJ (Clinical research ed.) 300, 1498-1499.

Armstrong, B.P., Simpson, H.K., Crouch, R., and Deakin, C.D. (2007). Prehospital clearance of the cervical spine: does it need to be a pain in the neck? Emerg. Med. J. 24, 501-503.

Benner, J.P., Brauning, G., Green, M., Caldwell, W., Borloz, M.P., and Brady, W.J. (2006). Disagreement between transport team and ED staff regarding the prehospital assessment of air medically evacuated scene patients. Air Med. J. 25, 165-169.

Brown, L.H., Gough, J.E., and Simonds, W.B. (1998). Can EMS providers adequately assess trauma patients for cervical spinal injury? Prehosp. Emerg. Care. 2, 33-36.
Burney, R.E., Waggoner, R., and Maynard, F.M. (1989). Stabilization of spinal injury for early transfer. J. Trauma 29, 1497-1499.

Burton, J.H., Dunn, M.G., Harmon, N.R., Hermanson, T.A., and Bradshaw, J.R. (2006). A statewide, prehospital emergency medical service selective patient spine immobilization protocol. J. Trauma 61, 161-167.

Burton, J.H., Harmon, N.R., Dunn, M.G., and Bradshaw, J.R. (2005). EMS provider findings and interventions with a statewide EMS spine-assessment protocol. Prehosp. Emerg. Care 9, 303-309.

Campbell, P. (1987). Comparison of flight nurses' prehospital assessments and emergency physicians' ED assessments of trauma patients. J. Emerg. Nurs. 13, 219-222.

Chan, D., Goldberg, R.M., Mason, J., and Chan, L. (1996). Backboard versus mattress splint immobilization: a comparison of symptoms generated. J. Emerg. Med. 14, 293-298.

Chandler, D.R., Nemejc, C., Adkins, R.H., and Waters, R.L. (1992). Emergency cervical-spine immobilization. Ann. Emerg. Med. 21, 1185-1188.

Cordell, W.H., Hollingsworth, J.C., Olinger, M.L., Stroman, S.J., and Nelson, D.R. (1995). Pain and tissue-interface pressures during spine-board immobilization. Ann. Emerg. Med. 26, 31-36.

Cornwell, E.E., 3rd, Chang, D.C., Bonar, J.P., Campbell, K.A., Phillips, J., Lipsett, P., Scalea, T., and Bass, R. (2001). Thoracolumbar immobilization for trauma patients with torso gunshot wounds: is it necessary? Arch. Surg. 136, 324-327.

Crosby, E.T. (2006). Airway management in adults after cervical spine trauma. Anesthesiology 104, 1293-1318.

Crosby, E.T. (1992). Tracheal intubation in the cervical spineinjured patient. Canadian J. Anaesthesia (J. canadien d'anesthesie.) 39, 105-109.

Davies, G., Deakin, C., and Wilson, A. (1996). The effect of a rigid collar on intracranial pressure. Injury 27, 647-649.

De Lorenzo, R.A., Olson, J.E., Boska, M., Johnston, R., Hamilton, G.C., Augustine, J., and Barton, R. (1996). Optimal positioning for cervical immobilization. Ann. Emerg. Med. 28, 301-308.

Domeier, R.M., Evans, R.W., Swor, R.A., Hancock, J.B., Fales, W., Krohmer, J., Frederiksen, S.M., and Shork, M.A. (1999). The reliability of prehospital clinical evaluation for potential spinal injury is not affected by the mechanism of injury. Prehosp. Emerg. Care 3, 332-337.

Domeier, R.M., Evans, R.W., Swor, R.A., Rivera-Rivera, E.J., and Frederiksen, S.M. (1997). Prospective validation of outof-hospital spinal clearance criteria: a preliminary report. Acad. Emerg. Med. 4, 643-646.

Domeier, R.M., Frederiksen, S.M., and Welch, K. (2005). Prospective performance assessment of an out-of-hospital protocol for selective spine immobilization using clinical spine clearance criteria. Ann. Emerg. Med. 46, 123-131.

Domeier, R.M., Swor, R.A., Evans, R.W., Hancock, J.B., Fales, W., Krohmer, J., Frederiksen, S.M., Rivera-Rivera, E.J., and Schork, M.A. (2002). Multicenter prospective validation of prehospital clinical spinal clearance criteria. J. Trauma 53, 744-750.

Doran, J.V., Tortella, B.J., Drivet, W.J., and Lavery, R.F. (1995). Factors influencing successful intubation in the prehospital setting. Prehosp. Disaster Med. 10, 259-264.

Downs, S.H., and Black, N. (1998). The feasibility of creating a checklist for the assessment of the methodological quality both of randomised and non-randomised studies of health care interventions. J. Epidemiol. Community Health 52, 377-384.

Eismont, F.J., Currier, B.L., and McGuire, R.A., Jr. (2004). Cervical spine and spinal cord injuries: recognition and treatment. Instructional Course Lectures 53, 341-358. 
Fehlings, M.G., and Louw, D. (1996). Initial stabilization and medical management of acute spinal cord injury. Am. Fam. Physician 54, 155-162.

Fenstermaker, R.A. (1993). Acute neurologic management of the patient with spinal cord injury. Urologic Clin. North Am. 20, 413-421.

Flabouris, A. (2001). Clinical features, patterns of referral and out of hospital transport events for patients with suspected isolated spinal injury. Injury 32, 569-575.

Gerling, M.C., Davis, D.P., Hamilton, R.S., Morris, G.F., Vilke, G.M., Garfin, S.R., and Hayden, S.R. (2000). Effects of cervical spine immobilization technique and laryngoscope blade selection on an unstable cervical spine in a cadaver model of intubation. Ann. Emerg. Med. 36, 293-300.

Graziano, A.F., Scheidel, E.A., Cline, J.R., and Baer, L.J. (1987). A radiographic comparison of prehospital cervical immobilization methods. Ann. Emerg. Med. 16, 1127-1131.

Hamilton, R.S., and Pons, P.T. (1996). The efficacy and comfort of full-body vacuum splints for cervical-spine immobilization. J. Emerg. Med. 14, 553-559.

Hasson, F., Keeney, S., and McKenna, H. (2000). Research guidelines for the Delphi survey technique. J. Adv. Nurs. 32, 1008-1015.

Hauswald, M., Hsu, M., and Stockoff, C. (2000). Maximizing comfort and minimizing ischemia: a comparison of four methods of spinal immobilization. Prehosp. Emerg. Care 4, 250-252.

Hauswald, M., Ong, G., Tandberg, D., and Omar, Z. (1998). Outof-hospital spinal immobilization: its effect on neurologic injury. Acad. Emerg. Med. 5, 214-219.

Huerta, C., Griffith, R., and Joyce, S.M. (1987). Cervical spine stabilization in pediatric patients: evaluation of current techniques. Ann. Emerg. Med. 16, 1121-1126.

Johnson, D.R., Hauswald, M., and Stockhoff, C. (1996). Comparison of a vacuum splint device to a rigid backboard for spinal immobilization. Am. J. Emerg. Med. 14, 369-372.

Keeney, S., Hasson, F., and McKenna, H.P. (2001). A critical review of the Delphi technique as a research methodology for nursing. Int. J. Nursing Studies 38, 195-200.

Kennedy, H.P. (2004). Enhancing Delphi research: methods and results. J. Adv. Nurs. 45, 504-511.

Krell, J.M., McCoy, M.S., Sparto, P.J., Fisher, G.L., Stoy, W.A., and Hostler, D.P. (2006). Comparison of the Ferno Scoop Stretcher with the long backboard for spinal immobilization. Prehosp. Emerg. Care 10, 46-51.

Luscombe, M.D., and Williams, J.L. (2003). Comparison of a long spinal board and vacuum mattress for spinal immobilisation. Emerg. Med. J. 20, 476-478.

Main, P.W., and Lovell, M.E. (1996). A review of seven support surfaces with emphasis on their protection of the spinally injured. J. Accident Emerg. Med. 13, 34-37.

Maruyama, K., Yamada, T., Kawakami, R., and Hara, K. (2008). Randomized cross-over comparison of cervical-spine motion with the AirWay Scope or Macintosh laryngoscope with in-line stabilization: a video-fluoroscopic study. Br. J. Anaesthesia 101, 563-567.

Mazolewski, P., and Manix, T.H. (1994). The effectiveness of strapping techniques in spinal immobilization. Ann. Emerg. Med. 23, 1290-1295.

McGuire, R.A., Neville, S., Green, B.A., and Watts, C. (1987). Spinal instability and the log-rolling maneuver. J. Trauma 27, 525-531.
Meldon, S.W., Brant, T.A., Cydulka, R.K., Collins, T.E., and Shade, B.R. (1998). Out-of-hospital cervical spine clearance: agreement between emergency medical technicians and emergency physicians. J. Trauma 45, 1058-1061.

Muhr, M.D., Seabrook, D.L., and Wittwer, L.K. (1999). Paramedic use of a spinal injury clearance algorithm reduces spinal immobilization in the out-of-hospital setting. Prehosp. Emerg. Care 3, 1-6.

Nypaver, M., and Treloar, D. (1994). Neutral cervical spine positioning in children. Ann. Emerg. Med. 23, 208-211.

Peery, C.A., Brice, J., and White, W.D. (2007). Prehospital spinal immobilization and the backboard quality assessment study. Prehosp. Emerg. Care. 11, 293-297.

Perry, S.D., McLellan, B., McIlroy, W.E., Maki, B.E., Schwartz, M., and Fernie, G.R. (1999). The efficacy of head immobilization techniques during simulated vehicle motion. Spine 24, 1839-1844.

Sackett, D.L., Strauss, S.E., Richardson, W.S., Rosenberg, W., and Haynes, R.B. (2000). Evidence-based medicine: How to practice and teach EBM. Toranto, Ontario: Churchill Livingstone.

Sahni, R., Menegazzi, J.J., and Mosesso, V.N., Jr. (1997). Paramedic evaluation of clinical indicators of cervical spinal injury. Prehosp. Emerg. Care 1, 16-18.

Scannell, G., Waxman, K., Tominaga, G., Barker, S., and Annas, C. (1993). Orotracheal intubation in trauma patients with cervical fractures. Arch. Surg. 128, 903-905; discussion 905-906.

Schafermeyer, R.W., Ribbeck, B.M., Gaskins, J., Thomason, S., Harlan, M., and Attkisson, A. (1991). Respiratory effects of spinal immobilization in children. Ann. Emerg. Med. 20, 10171019.

Schriger, D.L., Larmon, B., LeGassick, T., and Blinman, T. (1991). Spinal immobilization on a flat backboard: does it result in neutral position of the cervical spine? Ann. Emerg. Med. 20, 878-881.

Sheerin, F., and de Frein, R. (2007). The occipital and sacral pressures experienced by healthy volunteers under spinal immobilization: a trial of three surfaces. J. Emerg. Nurs. 33, 447-450.

Stroh, G., and Braude, D. (2001). Can an out-of-hospital cervical spine clearance protocol identify all patients with injuries? An argument for selective immobilization. Ann. Emerg. Med. 37, 609-615.

Turkstra, T.P., Craen, R.A., Pelz, D.M., and Gelb, A.W. (2005). Cervical spine motion: a fluoroscopic comparison during intubation with lighted stylet, GlideScope, and Macintosh laryngoscope. Anesthes. Analg. 101, 910-915, table of contents.

Walton, R., DeSalvo, J.F., Ernst, A.A., and Shahane, A. (1995). Padded vs. unpadded spine board for cervical spine immobilization. Acad. Emerg. Med. 2, 725-728.

Waninger, K.N., Richards, J.G., Pan, W.T., Shay, A.R., and Shindle, M.K. (2001). An evaluation of head movement in backboard-immobilized helmeted football, lacrosse, and ice hockey players. Clin. J. Sport Med. 11, 82-86.

Address correspondence to: Albert Yee, M.D., M.Sc., F.R.C.S.C. Department of Surgery University of Toronto 2075 Bayview Avenue Room MG-371

Toronto, Ontario M4N3M5, Canada

E-mail: Albert.yee@sunnybrook.ca 
